\title{
New Perspectives on the Generation and Maintenance of the Kuroshio Large Meander
}

\author{
YANG YANG \\ School of Marine Sciences, and Center for Ocean-Atmosphere Dynamical Studies, Nanjing University of \\ Information Science and Technology, Nanjing, China \\ X. SAN LIANG \\ School of Marine Sciences, and School of Atmospheric Sciences, and Center for Ocean-Atmosphere \\ Dynamical Studies, Nanjing University of Information Science and Technology, Nanjing, China
}

(Manuscript received 31 December 2018, in final form 30 May 2019)

\begin{abstract}
The internal dynamical processes underlying the Kuroshio large meander are investigated using a recently developed analysis tool, multiscale window transform (MWT), and the MWT-based canonical transfer theory. Oceanic fields are reconstructed on a low-frequency mean flow window, a mesoscale eddy window, and a high-frequency synoptic window with reference to the three typical path states south of Japan, that is, the typical large meander (tLM), nearshore non-large meander (nNLM), and offshore non-large meander (oNLM) path states. The interactions between the scale windows are quantitatively evaluated in terms of canonical transfer, which bears a Lie bracket form and conserves energy in the space of scale. In general, baroclinic (barotropic) instability is strengthened (weakened) during the tLM state. For the first time we found a spatially coherent inverse cascade of kinetic energy (KE) from the synoptic eddies to the slowly varying mean flow; it occupies the whole large meander region but exists only in the tLM state. By the timevarying multiscale energetics, a typical large meander is preceded by a strong influx of mesoscale eddy energy from upstream with a cyclonic eddy, which subsequently triggers a strong inverse KE cascade from the mesoscale window to the mean flow window to build up the KE reservoir for the meander. Synoptic frontal eddies are episodically intensified due to the baroclinic instability of the meander, but they immediately feed back to the mean flow window through inverse KE cascade. These results highlight the important role played by inverse KE cascades in generating and maintaining the Kuroshio large meander.
\end{abstract}

\section{Introduction}

As an intense western boundary current (WBC) in the North Pacific Ocean, the Kuroshio plays a key role in distributing momentum and heat in the northwest Pacific. It is well recognized that the Kuroshio south of Japan undergoes a unique bimodal path transition, oscillating between a large meander (LM) and a non-large meander (NLM) path state on the interannual time scale (Taft 1972; Kawabe 1985). During the LM (NLM) state, the Kuroshio off the Kii Peninsula tends to take a meandering (straight) path. The NLM path can be further divided into a nearshore NLM (nNLM) path and an offshore NLM (oNLM) path depending on whether the Kuroshio over the Izu-Ogasawara Ridge takes a northward or southward path (Kawabe 1995) (Fig. 1a).

Corresponding author: X. San Liang, sanliang@courant.nyu.edu
During the past few decades, continuing efforts have been made to understand the dynamics underlying the bimodal phenomenon. Generally, the bimodality could be either driven by external forcing or due to internal ocean dynamics. A number of numerical studies have considered wind forcing as an important factor for changing the Kuroshio path by controlling the strength of the upstream volume transport (Akitomo et al. 1996; Hurlburt et al. 1996; Kurogi and Akitomo 2003; Waseda et al. 2005; Tsujino et al. 2013). However, long-term observational time series indicate that the temporal variations of the Kuroshio's path state and its associated upstream transport are not well correlated after 1975 (Qiu and Miao 2000). In fact, some modeling studies have reported that multiple equilibrium regimes exist in the Kuroshio system even under climatological forcings (i.e., with no interannual atmospheric forcing), suggesting that the ocean's internal processes might be 

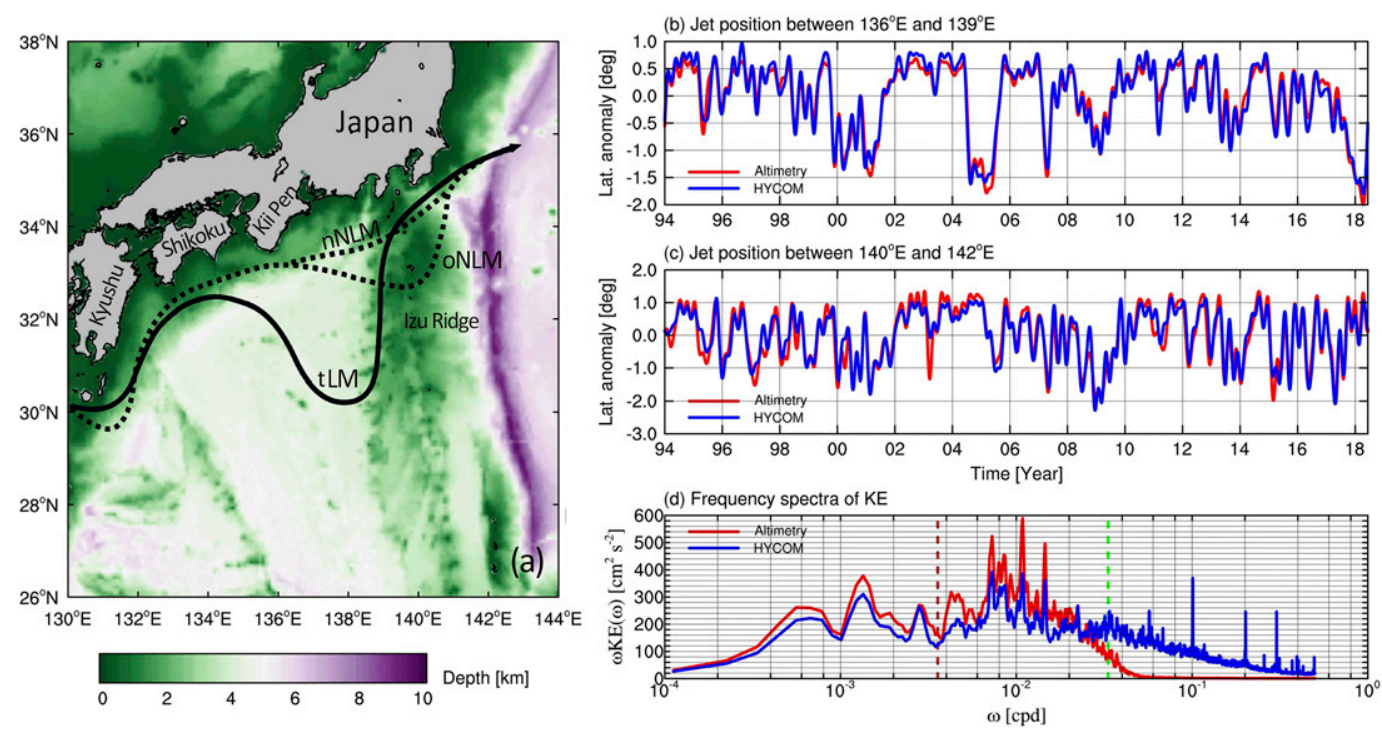

FIG. 1. (a) A schematic of the three typical paths (i.e., tLM, nNLM, and oNLM) of the Kuroshio south of Japan based on Kawabe (1995). Color shading denotes the bathymetry based on ETOPO1 dataset. (b) Jet position anomaly between $136^{\circ}$ and $139^{\circ} \mathrm{E}$ from the altimetry (red line) and HYCOM (blue line). The jet position is defined as the southernmost location of the Kuroshio axis between $136^{\circ}$ and $139^{\circ} \mathrm{E}$. (c) As in (b), but for the jet position anomaly between $140^{\circ}$ and $142^{\circ} \mathrm{E}$. (d) The variance-preserving frequency spectra KE averaged over the large meander region $\left(30^{\circ}-34^{\circ} \mathrm{N}, 135^{\circ}-140^{\circ} \mathrm{E}\right)$ from the altimetry (red line) and HYCOM (blue line). The dashed green and brown lines indicate the period of 30 and 280 days, respectively.

more important for the low-frequency bimodal variability (Hurlburt et al. 1996; Douglass et al. 2012; Kurogi et al. 2013; Wang et al. 2013).

Previous studies based on observations and numerical models have provided evidence that internal oceanic processes, such as baroclinic instability and eddy-mean flow interaction, are essential for the large meander formation (Yoon and Yasuda 1987; Qiu and Miao 2000; Endoh and Hibiya 2001; Miyazawa et al. 2004; Tsujino et al. 2006; Usui et al. 2008b; Tseng et al. 2012; Nagano et al. 2018). For example, it has been well established that the development of a typical LM (tLM) event is often triggered by a cold cyclonic eddy (i.e., the so-called trigger meander) propagating from upstream (Solomon 1978; Sekine 1990; Kawabe 1995; Miyazawa et al. 2008; Usui et al. 2008a; Liu et al. 2018) and amplified with an abyssal anticyclone around the Koshu Seamount (Waseda et al. 2003; Miyazawa et al. 2004; Tsujino et al. 2006; Usui et al. 2008b; Douglass et al. 2012). The vertical coupling between the deep eddies and the upper meander is found to be consistent with a baroclinic instability scenario (Endoh and Hibiya 2009; Tseng et al. 2012). It should be noted that these internal processes may be sensitive to the local continental geometry (Chao 1984; Yoon and Yasuda 1987; Hughes 1989; Yamagata and Umatani 1989) and bottom topographic features (Sekine 1990; Masuda and Akitomo 2000; Endoh et al. 2011; Mitsudera et al. 2006; Masuda 1982; Tanaka and Hibiya
2017), making the underlying dynamics more complex in this region.

Although as suggested in previous studies that baroclinic instability plays a key role in the LM formation south of Japan, barotropic instability may also significantly influence the meander through eddy-mean flow interactions (Yang and Liang 2016, 2019). Besides, recent studies have shown that nonlinear scale interactions can excite inverse energy cascades from high to low frequencies in the ocean (Berloff et al. 2007; Arbic et al. 2014; Sérazin et al. 2018), analogous to the transient eddy feedback mechanism in maintaining the low-frequency modes in the atmosphere (e.g., Holopainen 1978; Barnes and Hartmann 2010; Luo et al. 2015; Ma and Liang 2017). In this study, we employ a recently developed multiscale energetics analysis and canonical transfer theory (Liang 2016) to diagnose the energetics, especially the internal barotropic and baroclinic energy transfers among the mean flow, mesoscale eddies and high-frequency synoptic wave motions south of Japan, using outputs from the $1 / 12^{\circ}$ data-assimilating Hybrid Coordinate Ocean Model (HYCOM). We aim to clarify, in a quantitative way, the relative importance between intrinsic instabilities and nonlocal influences in triggering and maintaining the Kuroshio bimodality, and assess as well the role of inverse cascades, a mechanism which to our knowledge has not been addressed in the literature. The rest of the paper is organized as follows: we first describe the model and its validation in section 2, and then briefly 
introduce the methods in section 3. The major results are presented in sections 4 and 5. Section 6 summarizes this study.

\section{Data}

We use for this study the hindcast outputs from the U.S. Navy Global Ocean Forecasting System (GOFS), which includes HYCOM with Navy Coupled Ocean Data Assimilation (NCODA) system. HYCOM is an eddy-resolving ocean model with a horizontal resolution of $1 / 12^{\circ}$. It employs an adaptive vertical coordinate system with 32 hybrid vertical coordinate surfaces (Chassignet et al. 2007). The model is forced by 3-hourly $0.281^{\circ}$ atmospheric fields from Navy Global Environmental Model (NAVGEM) from the Fleet Numerical Meteorology and Oceanography Center. Using a $3 \mathrm{D}$ variational analysis scheme, the NCODA system assimilates available satellite-based altimeter, sea ice concentration and sea surface temperature observations, and in situ temperature and salinity profiles from XBTs, Argo floats, and moored buoys. A more thorough description of the model configuration can be found in Metzger et al. (2014, and the references therein). The model outputs are interpolated to the GLBv0.08 grid $\left(0.08^{\circ}\right.$ resolution between $40^{\circ} \mathrm{S}$ and $40^{\circ} \mathrm{N}, 0.04^{\circ}$ poleward of these latitudes) horizontally and to 40 standard fixed-depth levels vertically for public distribution. The daily outputs from January 1994 to June 2018 are used for this study.

Figure 1 provides the validation of the HYCOM outputs against the altimeter observations, which shows that HYCOM well captures the observed jet axis positions between $136^{\circ}$ and $139^{\circ} \mathrm{E}$ (Fig. 1b) and that between $140^{\circ}$ and $142^{\circ} \mathrm{E}$ (Fig. 1c). The jet position is defined as the southernmost location of the jet axis within a certain longitudinal band. The jet axis is defined as the $110-\mathrm{cm}$ sea surface height ( $\mathrm{SSH}$ ) contour for the altimeter observation, which is consistently located around the steepest gradients of SSH. For the HYCOM model, the $50-\mathrm{cm}$ contour is used to define the jet axis. The discrepancy between the definitions with these two datasets results from the different reference mean sea surfaces they use. But anyhow they agree well in reality. The time series of the jet position along the two longitudinal bands will be used as indices for the three typical path states of Kuroshio south of Japan (see section 3 for details). The high degree of fidelity of the HYCOM hindcast can be further demonstrated in the kinetic energy (KE) spectra averaged in the large meander domain (Fig. 1d); it captures almost all the observed spectral peaks in the mesoscale (30-280 day) as well as longer (i.e., interannual and longer) time scales, albeit with a slightly weaker amplitude compared to that of the observations. An advantage of HYCOM over the altimeter data is that HYCOM includes high-frequency synoptic motions (defined as processes with periods shorter than 30 days in this study) which are largely absent in the current gridded altimeter products because of inherent filtering of the satellite altimeter data (Arbic et al. 2014) (Fig. 1d). Oceanic synoptic processes within this frequency band such as high-frequency frontal Rossby waves contain a large amount of KE; they play an important role in scale interactions in the midlatitude ocean (Sérazin et al. 2018). The dynamical effect of these high-frequency variabilities on the mean flow and mesoscale field in different path states of the Kuroshio south of Japan is yet to be explored.

\section{Method}

Ever since Lorenz (1955) introduced the theory of energy cycle with a set of two-scale energy equations using Reynolds mean-perturbation decomposition, energetics analysis has become a powerful approach in the studies of eddy-mean flow interactions and flow instabilities (e.g., Liang and Robinson 2005, 2007; von Storch et al. 2012; Liang 2016). In this study, we apply a recently developed localized multiscale energetics analysis and the theory of canonical transfer to investigate the barotropic and baroclinic instabilities and internal scale-scale interactions in the Kuroshio system south of Japan. Several previous studies have already emphasized the importance of the two-way interaction between the mean flow and the eddies in the Kuroshio large meander formation by probing into the energetics using classical Lorenz-type formalism (Miyazawa et al. 2004; Tsujino et al. 2006; Usui et al. 2008b). However, using diagnostic tools that partition a field into a mean and an eddy part is difficult to distinguish among the relative contributions from motions with nonstationary background and those with over two time scales.

In this study, three ranges of scales or "scale windows," as called by Liang and Anderson (2007), are distinguished. On these scale windows respectively occur mean flow, mesoscale, and synoptic-scale processes. The scale partitioning is fulfilled using the multiscale window transform (MWT) developed by Liang and Anderson (2007), a functional analysis tool that decomposes a function space into a direct sum of orthogonal subspaces, each with an exclusive range of scales while having the local information retained. Such a subspace is termed a scale window, or simply a window. The MWT can be viewed as a generalization of the classical Reynolds decomposition; it was developed for a faithful representation of the multiscale energies (and any quadratic quantities) on the resulting scale windows, in order to make the analysis of multiscale energetics possible. Note that attempting to evaluate multiscale energies with the filtered (reconstructed) field variables is conceptually wrong (see appendix for a demonstration). In Liang and Anderson (2007), they established that, for 
TABLE 1. Multiscale energetic terms in Eqs. (1) and (2). The variable notations are conventional. For details, see Liang (2016).

\begin{tabular}{|c|c|c|}
\hline Symbol & Mathematical form & Meaning \\
\hline$K^{\pi}$ & $\frac{1}{2} \hat{\mathbf{v}}_{h}^{\sim \varpi} \cdot \hat{\mathbf{v}}_{h}^{\sim \varpi}$ & KE on scale window $\varpi$ \\
\hline $\mathbf{Q}_{K}^{\pi}$ & $\frac{1}{2}\left[\left(\widehat{\mathbf{v v}}_{h}\right)^{\sim \pi} \cdot \hat{\mathbf{v}}_{h}^{\sim \pi}\right]$ & Flux of KE on window $\varpi$ \\
\hline$\Gamma_{K}^{\pi}$ & $\frac{1}{2}\left[\left(\widehat{(\mathbf{v}}_{h}\right)^{\sim \pi}: \nabla^{\sim} \hat{\mathbf{v}}_{h}^{\sim \pi}-\nabla \cdot\left(\widehat{\mathbf{v}}_{h}\right)^{\sim \pi} \cdot \hat{\mathbf{v}}_{h}^{\sim \pi}\right]$ & Canonical transfer of KE to window $\varpi$ \\
\hline $\mathbf{Q}_{P}^{\pi}$ & $\frac{1}{\rho_{0}} \hat{\mathbf{v}}^{\sim \sigma} \hat{P}^{\sim \sigma}$ & Pressure flux on window $\varpi$ \\
\hline$b^{\pi}$ & $\frac{g_{g}^{g}}{\rho_{0}} \hat{\rho}^{\sim \pi} \hat{w}^{\sim \pi}$ & Buoyancy conversion on window $\varpi$ \\
\hline$A^{\varpi}$ & $\frac{1}{2} c\left(\hat{\rho}^{\sim \pi}\right)^{2}, \quad c=\frac{g^{2}}{\rho_{0}^{2} N^{2}}$ & APE on window $\varpi$ \\
\hline $\mathbf{Q}_{A}^{\pi}$ & $\frac{1}{2}\left[c \hat{\rho}^{\sim \pi} \widehat{(\mathbf{v} \rho)}^{\sim \pi}\right]$ & Flux of APE on window $\varpi$ \\
\hline $\begin{array}{l}\Gamma_{A}^{\sigma} \\
F_{K}^{w}, F_{A}^{\sigma}\end{array}$ & $\frac{c}{2}\left[\widehat{(\mathbf{v} \rho)}^{\sim \pi} \cdot \nabla \hat{\rho}^{\sim \pi}-\hat{\rho}^{\sim \pi} \nabla \cdot \widehat{(\mathbf{v} \rho)}^{\sim \pi}\right]$ & $\begin{array}{l}\text { Canonical transfer of APE to window } \varpi \\
\text { Dissipation (diffusion) of KE (APE) on window } \varpi \text { through subgrid } \\
\text { processes }\end{array}$ \\
\hline
\end{tabular}

some specially constructed orthogonal filters, there exists a transform-reconstruction pair, namely, MWT and its counterpart multiscale window reconstruction (MWR). In some sense MWR functions just like a filter in the traditional sense. What makes it different is that, for each MWR, there exists an MWT that gives coefficients which can be used to represent the energy of the filtered series.

In this study, the mesoscale window is chosen to be bounded by cutoff periods of 280 days and 30 days (see Fig. 1d), following the previous eddy characteristics studies of this region (Ebuchi and Hanawa 2000). Processes with periods longer than 280 days are defined as the slowly varying mean flow; it includes the low-frequency transitions of the Kuroshio path states, that is, the interannual large meandering processes. The residual high-frequency signals with periods shorter than 30 days are treated as synoptic variabilities. We have also tried 15 days as the synoptic-scale window bound, and the results are similar. For easy reference, the three windows are denoted by $\varpi=$ 0,1 , and 2 , respectively. In the following, we present a brief introduction of the MWT-based multiscale energetics framework and canonical transfer theory. Details, including some major differences from the classical formalisms, are provided in the appendix.

Using MWT, Liang (2016) derived from primitive equations the governing equations for the multiscale $\mathrm{KE}$ and available potential energy (APE):

$$
\begin{aligned}
& \frac{\partial K^{\varpi}}{\partial t}+\nabla \cdot \mathbf{Q}_{K}^{\varpi}=\Gamma_{K}^{\varpi}-\nabla \cdot \mathbf{Q}_{P}^{\varpi}+b^{\varpi}+F_{K}^{\varpi}, \quad \text { and } \\
& \frac{\partial A^{\varpi}}{\partial t}+\nabla \cdot \mathbf{Q}_{A}^{\varpi}=\Gamma_{A}^{\varpi}-b^{\varpi}+F_{A}^{\varpi},
\end{aligned}
$$

for each scale window $\varpi=0,1,2$, where the expressions and meanings for the symbols are listed in Table 1. Note that all the terms in Eqs. (1) and (2) are four-dimensional field variables; in other words, they are all localized in space and time, distinctly different from the classical Lorenz-type formalisms (e.g., Lorenz 1955; von Storch et al. 2012) in which localization is lost in at least one dimension of space-time to achieve the scale decomposition.

Another aspect which distinguishes the above formalism from the classical ones is that the cross-scale transfer term (i.e., $\Gamma_{K}^{\pi}$ and $\Gamma_{A}^{\pi}$ ) conserves energy in the space of scale, that is, it satisfies the following property

$$
\sum_{\pi} \sum_{n} \Gamma_{n}^{\varpi}=0
$$

where $\sum_{\pi}$ and $\sum_{n}$ are the summation over all the scale windows $\varpi$ and sampling time steps $n$, respectively. [The subscript $n$ is omitted in Eqs. (1) and (2) for simplicity]. This is done by a careful separation of intertwined nonlinear processes into a transport and a transfer (Liang 2016). Physically, a transport process (i.e., $\nabla \cdot \mathbf{Q}_{K}^{\sigma}$ and $\left.\nabla \cdot \mathbf{Q}_{A}^{\sigma}\right)$ only redistributes energy in physical space and hence integrates to zero over a closed domain, while a transfer process is a redistribution of energy among the scale windows, without generating or destroying energy as a whole. The latter property, which seems to be a natural requirement, however does not hold in other energetics formalisms [see Liang (2016) for a comprehensive review]. To distinguish, this process is termed "canonical transfer." Besides, it has an expression in a Lie bracket form that satisfies the Jacobian identity, reminiscent of the Poisson bracket in Hamiltonian mechanics. More details of the canonical transfer are provided in the appendix.

As is well known in geophysical fluid dynamics, barotropic and baroclinic instabilities can be quantitatively described by the interscale transfers of the KE and APE between the mean flow and the fluctuations, respectively (e.g., Pedlosky 1987). As proved by Liang (2016), the $\Gamma_{K}^{\varpi}$ 

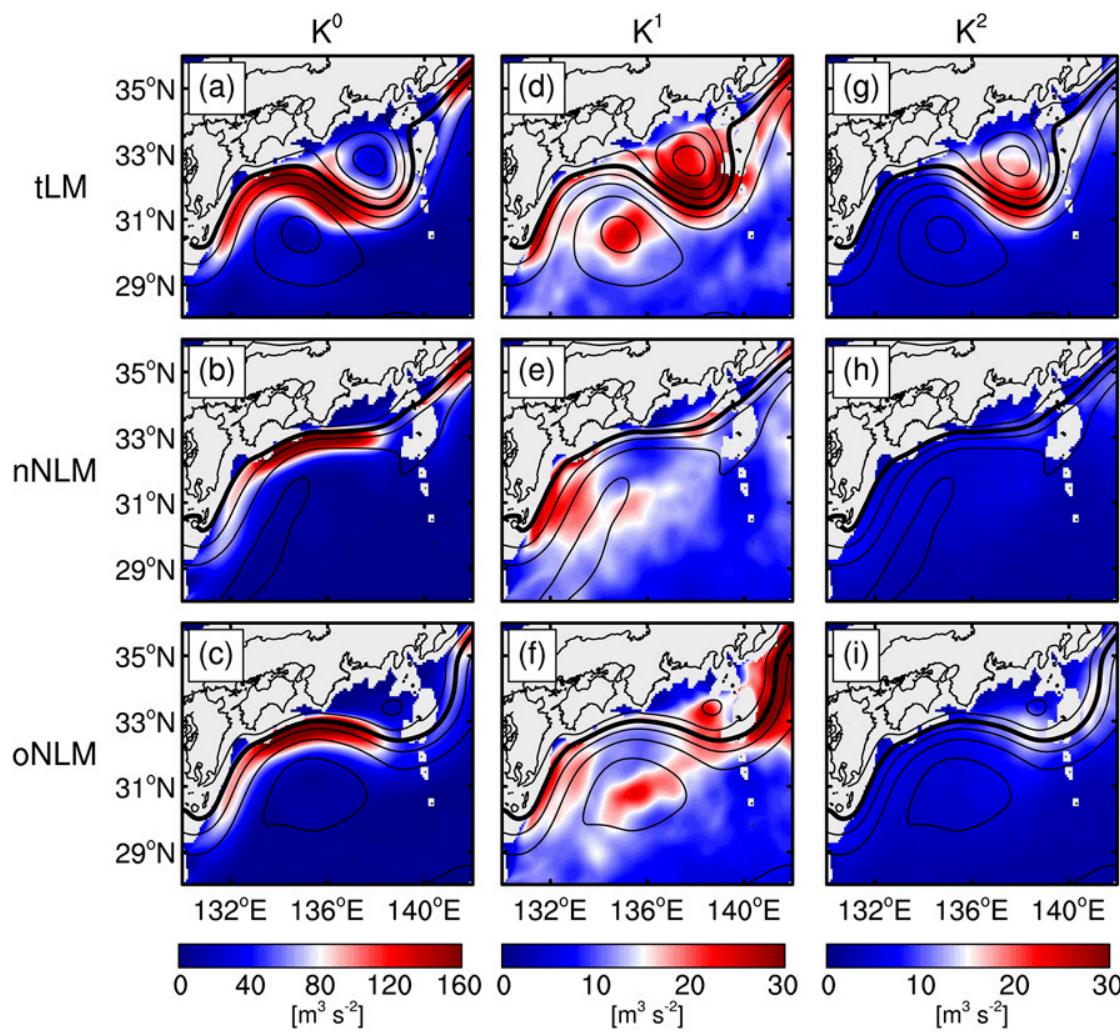

FIG. 2. Composite maps of the depth-integrated (upper $1000 \mathrm{~m}$ ) KE components $\left(\mathrm{m}^{3} \mathrm{~s}^{-2}\right)$ for the (a),(d),(g) tLM state, (b),(e),(h) nNLM state, and (c),(f),(i) oNLM state. The composite sea surface height field in each state is superposed in each figure, with the thick black line indicating the $50-\mathrm{cm}$ contour. The contour interval is $10 \mathrm{~cm}$.

and $\Gamma_{A}^{m}$ correspond precisely to the barotropic instability and baroclinic instability, respectively. By applying a three-scale window decomposition, we are able to quantify the intricate mutual interactions between different scales. For convenience, we use the superscript $\varpi_{0} \rightarrow \varpi_{1}$ to signify the interscale energy transfer. For instance, the canonical transfer of KE (APE) from the mean flow window to the mesoscale window is denoted as $\Gamma_{K}^{0 \rightarrow 1}\left(\Gamma_{A}^{0 \rightarrow 1}\right)$. A positive $\Gamma_{K}^{0 \rightarrow 1}\left(\Gamma_{A}^{0 \rightarrow 1}\right)$ is indicative of barotropic (baroclinic) instability. In this sense, these two transfers are also referred to as barotropic transfer and baroclinic transfer, respectively.

In the next section, composite analysis is used to examine the above canonical transfers and other relevant energy terms in the three typical path states of the Kuroshio south of Japan. We select the three composites based on two indices which reflect the southernmost positions of the Kuroshio axis between $136^{\circ}$ and $139^{\circ} \mathrm{E}$ (denoted as Index 1, see Fig. 1b), and between $140^{\circ}$ and $142^{\circ} \mathrm{E}$ (denoted as Index 2, see Fig. 1c), respectively. A tLM state occurs when Index 1 has a value lower than $32^{\circ} \mathrm{N}$ while Index 2 has a value greater than $33^{\circ} \mathrm{N}$. An nNLM state occurs when both indices are greater than $33^{\circ} \mathrm{N}$. An oNLM state occurs when Index 1 has a value greater than $32^{\circ} \mathrm{N}$ while Index 2 has a value lower than $33^{\circ} \mathrm{N}$. According to the definition above, 1162,1682 and 1319 days are selected for the LM, nNLM, and oNLM composites, respectively, during January 1994-June 2018.

\section{Composition of the energetics in the three typical path states}

\section{a. Multiscale energies}

Figure 2 shows the horizontal patterns of the multiscale KE components averaged over the respective periods of the three composite states. The mean flow KE $\left(K^{0}\right)$ depicts the slowly varying Kuroshio jet, which appears strengthened (weakened) and broadened (narrowed) in the LM (NLM) state (Figs. 2a-c). When averaged over the large meander domain $\left(30^{\circ}-34^{\circ} \mathrm{N}\right.$, $\left.135^{\circ}-140^{\circ} \mathrm{E}\right)$, the area-mean $K^{0}$ is much larger in the tLM state $\left(65.42 \mathrm{~m}^{3} \mathrm{~s}^{-2}\right)$ than the nNLM state $\left(30.0 \mathrm{~m}^{3} \mathrm{~s}^{-2}\right)$, or the oNLM state $\left(38.45 \mathrm{~m}^{3} \mathrm{~s}^{-2}\right)$ (Table 2), indicating that the background transport is large (small) when the Kuroshio south of Japan takes a meander (straight) path, consistent with previous studies (Chao 1984; Qiu and Miao 2000). The mesoscale eddy $\operatorname{KE~}\left(K^{1}\right)$ exhibits 
TABLE 2. Area-mean KE components $\left(\mathrm{m}^{3} \mathrm{~s}^{-2}\right)$ and canonical transfers $\left(10^{-6} \mathrm{~m}^{3} \mathrm{~s}^{-3}\right)$ in the large meander region south of Japan $\left(30^{\circ}-\right.$ $\left.34^{\circ} \mathrm{N}, 135^{\circ}-140^{\circ} \mathrm{E}\right)$ for the tLM, nNLM, and oNLM states.

\begin{tabular}{lcccccrrr}
\hline \hline & $K^{0}$ & $K^{1}$ & $K^{2}$ & $\Gamma_{A}^{0 \rightarrow 1}$ & $\Gamma_{A}^{0 \rightarrow 2}$ & $\Gamma_{A}^{1 \rightarrow 2}$ & $\Gamma_{K}^{0 \rightarrow 1}$ & \multicolumn{1}{c}{$\Gamma_{K}^{0 \rightarrow 2}$} \\
\hline tLM & 65.42 & 17.19 & 11.82 & 8.48 & 8.50 & 1.46 & 3.97 & -9.99 \\
nNLM & 30.00 & 10.45 & 4.70 & 2.47 & 1.19 & 0.95 & 10.23 & 1.42 \\
oNLM & 38.45 & 12.96 & 6.54 & 4.83 & 2.46 & 0.94 & 7.21 & 0.82 \\
\hline
\end{tabular}

elevated values off the Kii Peninsula during the tLM state, which is in good agreement with the large meander in this state, while its magnitude is greatly reduced during the other two NLM states (Figs. 2d-f and Table 2). Although the synoptic-scale window $\left(K^{2}\right)$ contains less KE than the other two lower-frequency windows in the two NLM states, it can be quite large in the tLM state $\left(11.82 \mathrm{~m}^{3} \mathrm{~s}^{-2}\right.$ ) (Figs. $2 \mathrm{~g}-\mathrm{i}$ and Table 2). The above analysis of the multiscale KE components reveals that, when the meander is strong (weak), the Kuroshio south of Japan contains more (less) energy in all three frequency bands. This implies that stronger (weaker) scale interactions may occur in the LM (NLM) state. It is interesting to note that, when the Kuroshio takes the oNLM path, there are more mesoscale activities on the eastern side of Izu-Ogasawara Ridge where the Kuroshio Extension is originated (Fig. 2f). Previous studies have shown that the Kuroshio
Extension tends to take a meandering path (leading to elevated regional EKE level) when the Kuroshio takes an oNLM path (e.g., Sugimoto and Hanawa 2012).

\section{b. Baroclinic canonical transfers}

We first analyze the baroclinic canonical transfers among the three scale windows. Figure 3 presents the horizontal maps of the depth-integrated transfers $\Gamma_{A}^{0 \rightarrow 1}$, $\Gamma_{A}^{0 \rightarrow 2}$, and $\Gamma_{A}^{1 \rightarrow 2}$ composited for the three path states. A general observation is that, although mingled with some negative spots (such as at the southern tip of Shikoku and on the western side of Izu-Ogasawara Ridge), the baroclinic transfers are overall positive in this region. That is to say, the canonical transfers of APE are mainly downscale, consistent with the baroclinic instability observed in previous studies (Tulloch et al. 2011). Intense positive $\Gamma_{A}^{0 \rightarrow 1}$ centers are distributed in the regions
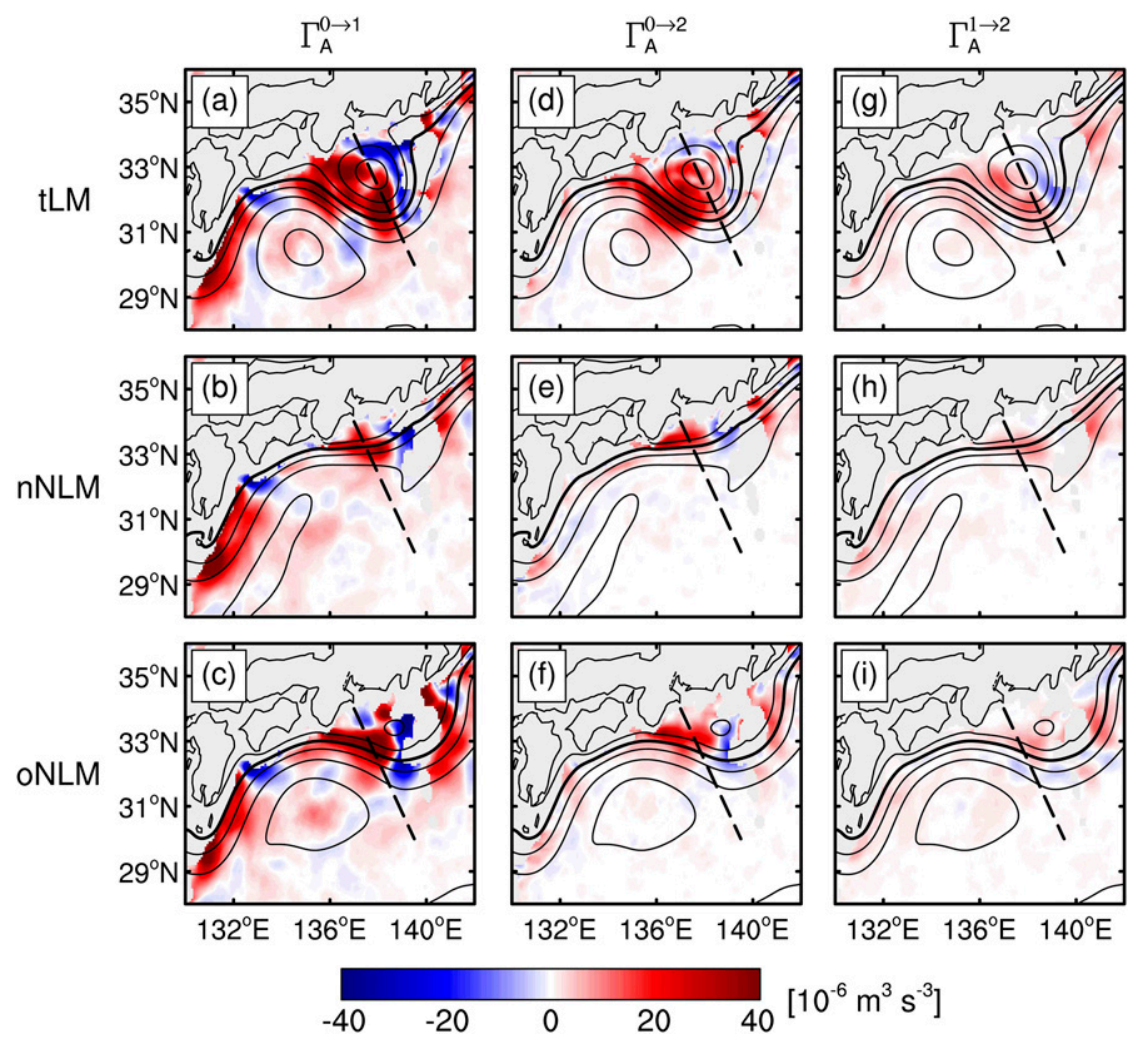

FIG. 3. As in Fig. 2, but for the baroclinic canonical transfers $\left(10^{-6} \mathrm{~m}^{3} \mathrm{~s}^{-3}\right)$. The dashed line in each figure denotes the cross section, which will be examined in Fig. 6. 


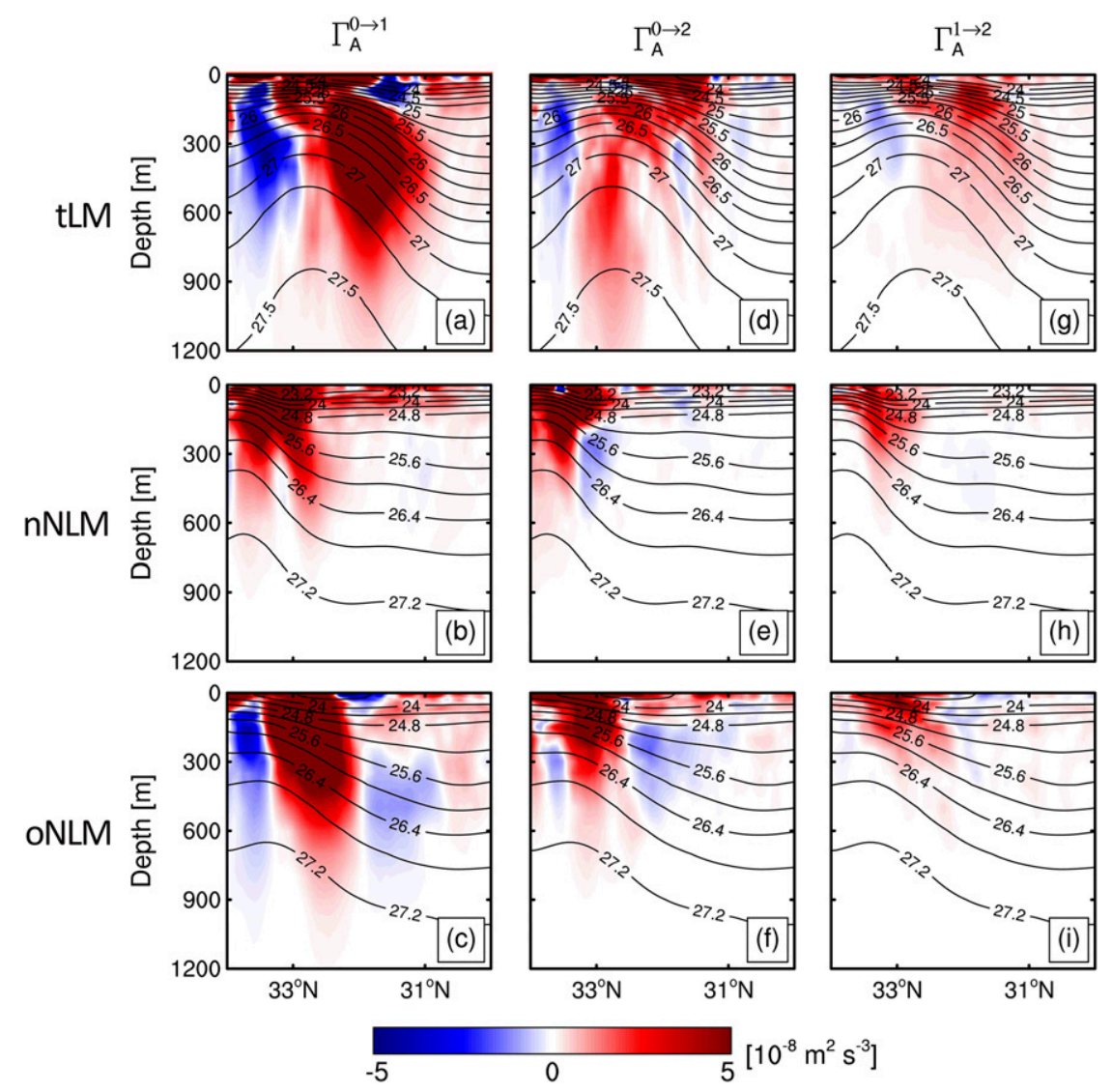

FIG. 4. The vertical structures of the baroclinic canonical transfers $\left(10^{-8} \mathrm{~m}^{2} \mathrm{~s}^{-3}\right)$ for the (a),(d),(g) tLM state, (b),(e),(h) nNLM state, and (c),(f),(i) oNLM state across the section as indicated in Fig. 3. The composite potential density $\left(\mathrm{kg} \mathrm{m}^{-3}\right)$ in each state is superposed in each figure.

south of Kyushu and southeast of Kii Peninsula in all three states, indicating that the Kuroshio is persistently baroclinically unstable in these regions (Figs. 3a-c). During the tLM state, the positive $\Gamma_{A}^{0 \rightarrow 1}$ off the Kii Peninsula is much stronger compared to that during the other two NLM states. Besides, the shape of the positive baroclinic transfer center is more spread out in the tLM state, in good agreement with the large meander in this state. The area-mean $\Gamma_{A}^{0 \rightarrow 1}$ reaches $8.8 \times 10^{-6} \mathrm{~m}^{3} \mathrm{~s}^{-3}$ during the tLM state, nearly 4 (2) times larger than that in the nNLM (oNLM) state (Table 2). This suggests the active role played by the large meander to elevate the regional baroclinic instability, as also observed in previous studies (e.g., Miyazawa et al. 2004; Tsujino et al. 2006; Tanaka and Hibiya 2017). Figures 6a-c further show the vertical structure of the $\Gamma_{A}^{0 \rightarrow 1}$ and potential density across the section normal to the Japan coastline (indicated in Fig. 3). One can see that, during the tLM state, strong and deep-reaching downscale $\Gamma_{A}^{0 \rightarrow 1}$ is concentrated along the jet axis around $32^{\circ} \mathrm{N}$, while weak and upscale $\Gamma_{A}^{0 \rightarrow 1}$ is found on the inshore side of the jet. In contrast,
$\Gamma_{A}^{0 \rightarrow 1}$ is significantly reduced in magnitude and only confined in the upper 600-m water column in the nNLM state. The oNLM state is also characterized by strong baroclinic instability around $33^{\circ} \mathrm{N}$ but the baroclinic instability is relatively weaker than that in the tLM state (Fig. 4c).

We now examine the interaction between the synoptic eddies and mean flow, and that between the synoptic eddies and mesoscale eddies. This is made possible thanks to HYCOM's high temporal resolution. A welldefined positive center of $\Gamma_{A}^{0 \rightarrow 2}$ is distributed south of Kii Peninsula for the tLM state, which coincides with the maximum of the synoptic-scale KE (Fig. 3d), implying that the high-frequency variabilities are generated through the baroclinic instability of the mean flow. This is in contrast to the two NLM states, during which the mean flow shifts northward, leaving a small area of weak downscale baroclinic canonical transfer there (Figs. 3e,f and Figs. 4e,f). The baroclinic transfer between the mesoscale eddies and synoptic-scale eddies (i.e., $\Gamma_{A}^{1 \rightarrow 2}$ ) is generally small compared to the other two baroclinic transfers and its strength is mainly confined in the upper 

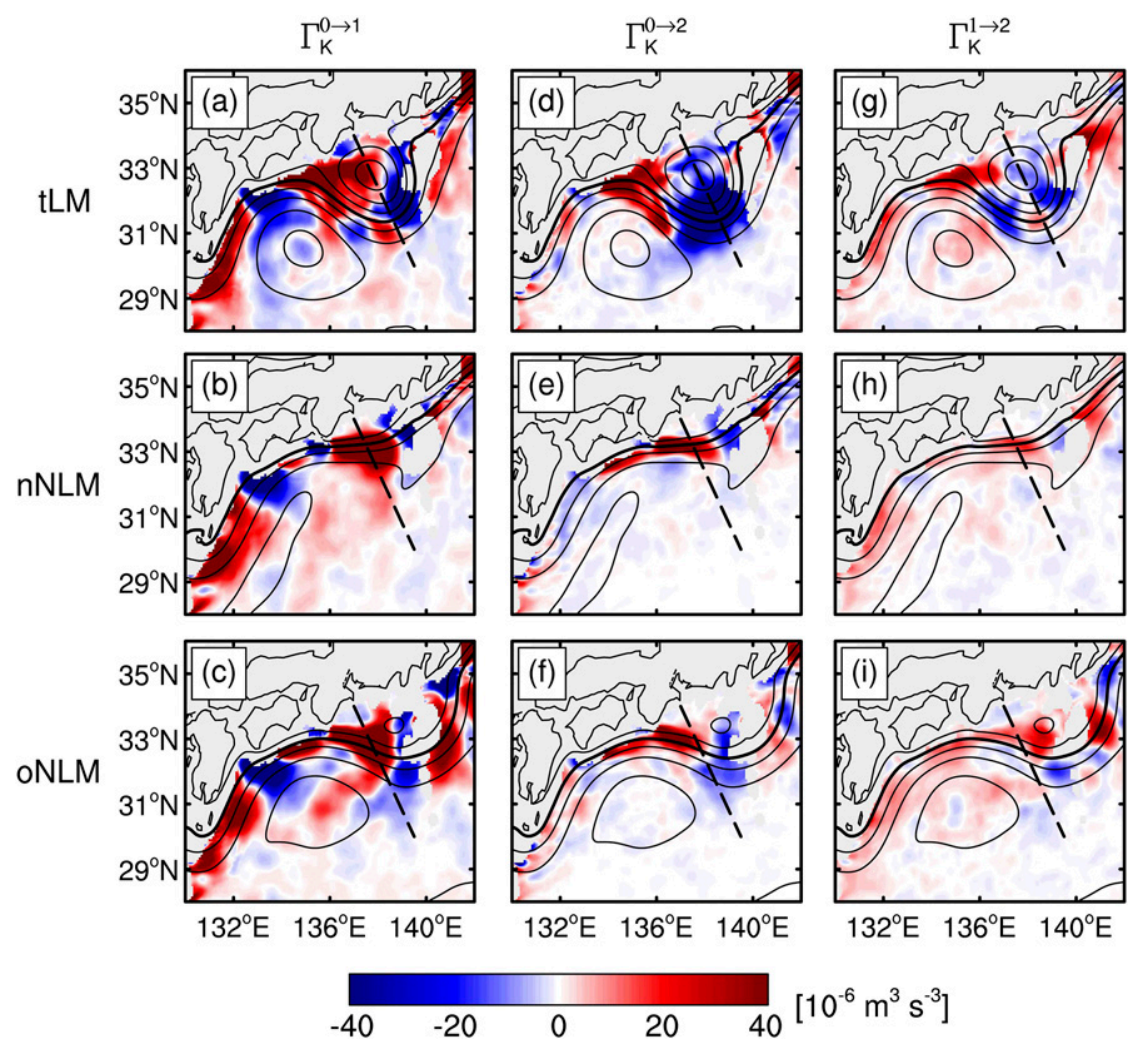

FIG. 5. As in Fig. 3, but for the barotropic canonical transfers.

500-m depth (Figs. 3g-i, Figs. 4g-i, and Table 2). Similar to $\Gamma_{A}^{0 \rightarrow 1}$ and $\Gamma_{A}^{0 \rightarrow 2}$, this term is stronger during the tLM state than that during the two NLM states, indicating that the forward APE cascade from mesoscale eddy field is also an energy source for the elevated synoptic-scale eddy activity in the tLM state.

\section{c. Barotropic canonical transfers}

Previous modeling studies have shown that the Kuroshio's path transitions can also arise in simple barotropic models (Chao 1984; Yasuda et al. 1985; Akitomo et al. 1991; Schmeits and Dijkstra 2001; Wang et al. 2013). This implies that barotropic instability may also play an important role in modulating such lowfrequency variations (Pierini et al. 2014). To see whether this is true, in the following we examine the barotropic canonical transfers among the mean flow, mesoscale eddies, and synoptic motions (i.e., $\Gamma_{K}^{0 \rightarrow 1}, \Gamma_{K}^{0 \rightarrow 2}$, and $\Gamma_{K}^{1 \rightarrow 2}$ ) for the three path states.

Figure 5 shows the composite patterns of depthintegrated barotropic canonical transfers averaged respectively over the periods of the three path states. In contrast to the baroclinic transfers whose sign is mainly positive, the barotropic transfer terms generally exhibit a train of positive and negative patches along the Japan coast. Strong forward barotropic transfer from the mean flow to the mesoscale eddies (i.e., $\Gamma_{K}^{0 \rightarrow 1}$ ) is observed in the region south of Kyushu as well as southeast of Kii Peninsula in all three composite states (Figs. 5a-c). In other words, the Kuroshio is constantly barotropically unstable in these two regions. During the tLM state, positive $\Gamma_{K}^{0 \rightarrow 1}$ occupies the western part of the meander trough, indicating that the large meander loses KE to the mesoscale eddies through barotropic instability (Fig. 5a). On the eastern side of the trough, the mean flow extracts $\mathrm{KE}$ from the mesoscale eddies via strong inverse KE cascade, indicating that mesoscale eddies tend to drive the large meander in this region (Figs. 5a and 6a). A similar phenomenon has also been suggested to potentially influence the low-frequency variability of background jet in the Kuroshio Extension region (e.g., Pierini 2006; Qiu and Chen 2010; Gentile et al. 2018). In contrast, during the nNLM state, the region off the Kii Peninsula is dominated by a single positive $\Gamma_{K}^{0 \rightarrow 1}$ center (Fig. 5b), with a mean transfer rate of $10.23 \times$ $10^{-6} \mathrm{~m}^{3} \mathrm{~s}^{-3}$ over the large meander region, much larger than the rate of $3.97 \times 10^{-6} \mathrm{~m}^{3} \mathrm{~s}^{-3}$ for the tLM state (Table 2). To compare the strengths of the barotropic instabilities in these states, we also calculate the mean transfer rate averaged over a relatively small area where 

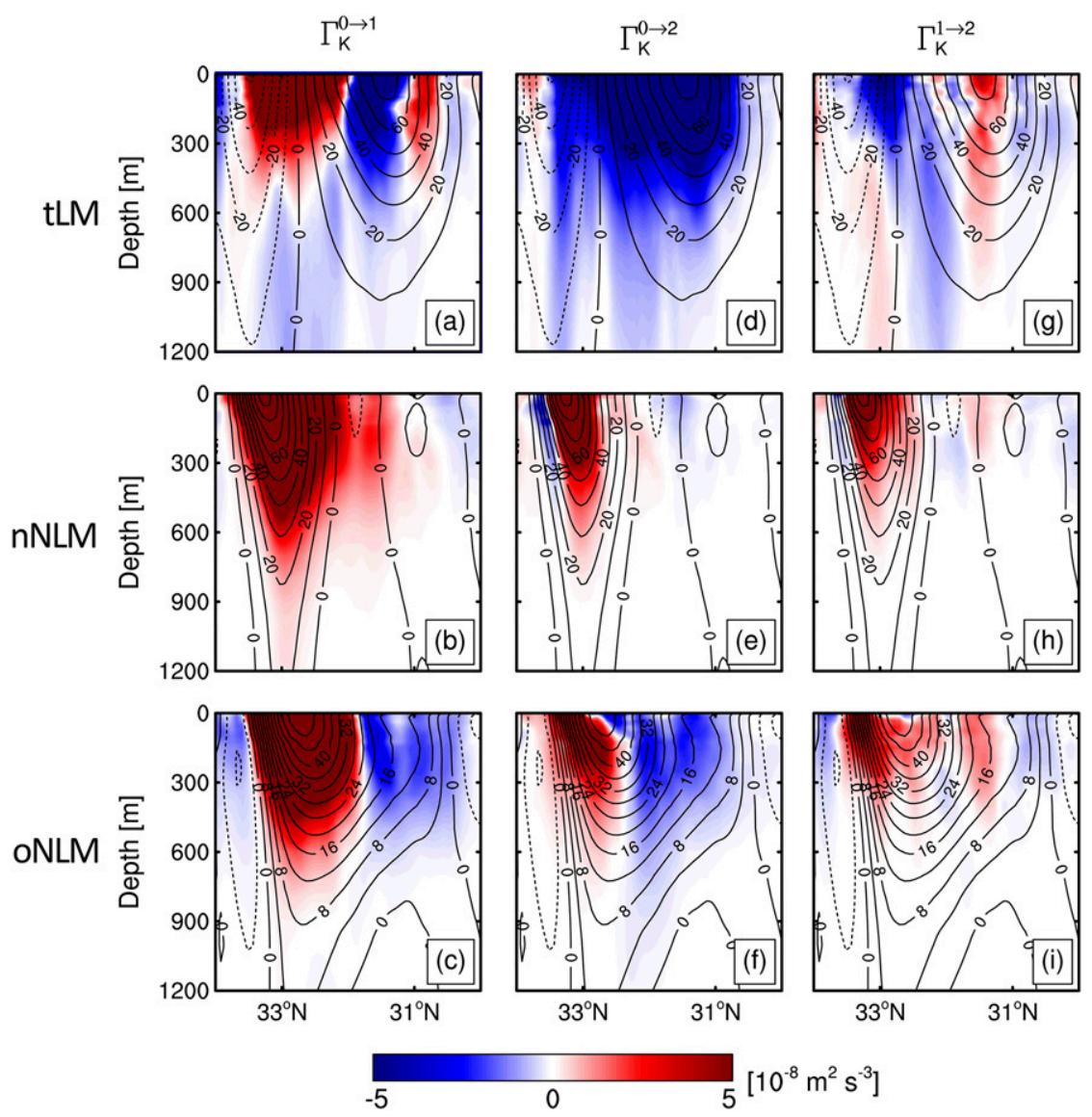

FIG. 6. As in Fig. 4, but for the barotropic canonical transfers. The composite current velocity normal to the section $\left(\mathrm{cm} \mathrm{s}^{-1}\right)$ in each state is superposed in each figure.

only positive $\Gamma_{K}^{0 \rightarrow 1}$ spots are covered. The result shows that the Kuroshio in this region becomes more barotropically unstable in the nNLM state $(42.33 \times$ $\left.10^{-6} \mathrm{~m}^{3} \mathrm{~s}^{-3}\right)$ than in the tLM state $\left(26.07 \times 10^{-6} \mathrm{~m}^{3} \mathrm{~s}^{-3}\right)$. The above analysis shows that the strength of barotropic instability tends to be reduced when the Kuroshio takes a meandering path; meanwhile, on the eastern side of the meander trough, mesoscale eddies act to strengthen the meander via significant inverse KE cascades.

The barotropic canonical transfer $\Gamma_{K}^{0 \rightarrow 2}$ has a different horizontal and vertical distribution. During the tLM state, the most conspicuous feature is the overwhelming inverse $\mathrm{KE}$ cascade from the synoptic eddies to the mean flow (Figs. $5 \mathrm{~d}$ and $6 \mathrm{~d}$ ), with a mean transfer rate of $-9.99 \times 10^{-6} \mathrm{~m}^{3} \mathrm{~s}^{-3}$ in the large meander region (Table 2). Such an inverse KE cascade phenomenon is not found in the nNLM state when the Kuroshio takes a straight path (Figs. 5e,f, Figs. 6e,f, and Table 2). This indicates that the high-frequency eddies play an important role in maintaining the large meander. Figures $5 \mathrm{~g}-\mathrm{i}$ and Figs. 6g-i illustrate the spatial structures of the barotropic transfer underlying the eddy-eddy interactions between the synoptic-scale eddies and the mesoscale eddies (i.e., $\Gamma_{K}^{1 \rightarrow 2}$ ). Significant negative centers are distributed in the meander region during the tLM phase, suggesting that the high-frequency synoptic motions feed the lowerfrequency mesoscale eddy field via inverse temporal $\mathrm{KE}$ cascade. The temporal KE cascade is mainly forward during the nNLM and oNLM states, demonstrating the important role of high-frequency eddies in the multiscale interaction of the large meander event. Recently, there is a growing interest in assessing the characteristics of the temporal inverse cascades of $\mathrm{KE}$ in the ocean (e.g., Arbic et al. 2014; O'Rourke et al. 2018; Sérazin et al. 2018; Gentile et al. 2018). For instance, using high-resolution models, Sérazin et al. (2018) found that high-frequency geostrophic structures such as frontal Rossby waves act to feed low-frequency variabilities through nonlinear temporal inverse cascades in midlatitude ocean. They also emphasized that such inverse cascade could be disguised in the current altimetry observations due to spatial and temporal filtering inherent in the altimeter product. 
TABLE 3. As in Table 2, but for the energy budget terms on the mean flow window $(\varpi=0)\left(10^{-6} \mathrm{~m}^{3} \mathrm{~s}^{-3}\right)$.

\begin{tabular}{lrrrrr}
\hline \hline Term & $\partial E^{0} / \partial t$ & \multicolumn{1}{c}{$\Gamma_{A}^{0}$} & \multicolumn{1}{c}{$\Gamma_{K}^{0}$} & $\nabla \cdot \mathbf{Q}_{E}^{0}$ & Residue \\
\hline tLM & 4.78 & -13.99 & 5.83 & -71.91 & -58.97 \\
nNLM & -1.13 & -4.64 & -10.41 & -42.85 & -28.93 \\
oNLM & 0.01 & -7.17 & -6.66 & -54.71 & -40.87 \\
\hline
\end{tabular}

\section{d. Energy budgets}

It should be noted that scale interactions are only part of the complete energy cycle [Eqs. (1) and (2)]. To better understand the processes underlying the Kuroshio large meander, in this subsection, we estimate the mechanical energy budget by summing Eqs. (1) and (2),

$$
\frac{\partial E^{\varpi}}{\partial t}+\nabla \cdot \mathbf{Q}_{E}^{\varpi}=\Gamma_{K}^{\varpi}+\Gamma_{A}^{\varpi}-\nabla \cdot \mathbf{Q}_{P}^{\varpi}+F_{E}^{\varpi},
$$

where $\nabla \cdot \mathbf{Q}_{E}^{\Phi}$ and $F_{E}^{\Phi}$ are transport and dissipation of mechanical energy on window $\varpi\left(E^{\varpi}=A^{\varpi}+K^{\varpi}\right)$. Here, $\nabla \cdot \mathbf{Q}_{E}^{\varpi}$ is dominated by $\nabla \cdot \mathbf{Q}_{K}^{\varpi}$ (not shown), and positive (negative) values of $\nabla \cdot \mathbf{Q}_{E}^{m}$ represent the energy transported out of (into) the local domain. We found that the pressure work term $\left(\nabla \cdot \mathbf{Q}_{P}^{\varpi}\right)$ estimated from the data exhibits unrealistically noisy and large-amplitude structures. This is probably caused by the fact that considerable errors may come into the evaluation of the pressure field (which is not directly available from the HYCOM outputs) from the hydrostatic relation. For this reason, in the following, we will treat the last two terms on the right hand side of Eq. (4) as a single residue term. Note that diagnoses of the complete energy cycle is more suitable using free-running models or models with dynamically consistent assimilation schemes to conserve energy (Wunsch et al. 2009), which is beyond the scope of the present study.

Tables 3-5 give the composited mean values of the budget terms on windows 0,1 , and 2 , respectively. On the mean flow window, the energy transported from surrounding regions into the large meander region dominates the local energy balance (Table 3 ). It is clear that more $E^{0}$ is transported into this region during the tLM state than the other two NLM states. To see how energy is transported horizontally, we plot in Fig. 7 the horizontal vectors of the energy fluxes (i.e., $\mathbf{Q}_{E}^{\varpi}$ ) on the three scale windows. Clearly, the $\mathbf{Q}_{E}^{\varpi}$ vectors follow the mean current in each state, indicating that the transport is dominated by energy advection by the mean Kuroshio jet. In all three states, the incoming $E^{0}$ transported from the upstream region is mostly balanced by the residue term (i.e., pressure work and dissipation processes) (Table 3). It is interesting to note that the canonical KE transfer $\Gamma_{K}^{0}$ is another important contributor
TABLE 4. As in Table 2, but for the energy budget terms on the mesoscale window $(\varpi=1)\left(10^{-6} \mathrm{~m}^{3} \mathrm{~s}^{-3}\right)$.

\begin{tabular}{lrrrrr}
\hline \hline Term & $\partial E^{1} / \partial t$ & $\Gamma_{A}^{1}$ & $\Gamma_{K}^{1}$ & $\nabla \cdot \mathbf{Q}_{E}^{1}$ & Residue \\
\hline tLM & -1.34 & 6.64 & 2.94 & -1.42 & -12.34 \\
nNLM & 0.20 & 1.92 & 9.62 & 2.88 & -8.46 \\
oNLM & -0.70 & 4.62 & 6.35 & 1.61 & -10.06 \\
\hline
\end{tabular}

for the positive $E^{0}$ tendency in the tLM state due to upscale KE cascades. By contrast, $\Gamma_{K}^{0}$ shows negative values in the two NLM states (Table 3), meaning that the mean flow loses KE to the other scale windows via barotropic instability when the Kuroshio takes straight paths. On the mesoscale window, $\Gamma_{A}^{1}, \Gamma_{K}^{1}$, and the residue are dominant processes (Table 4). Both $\Gamma_{A}^{1}$ and $\Gamma_{K}^{1}$ make positive contributions to the mesoscale energy budget, with their relative roles varying in different states. Specifically, in the tLM state, $\Gamma_{A}^{1}$ is the major energy source for the mesoscale eddies in the large meander region, whereas in the nNLM state $\Gamma_{K}^{1}$ prevails, and $\Gamma_{A}^{1}$ is suppressed. A similar seesaw relation about the mixed instability (barotropic and baroclinic instability) energetics is also found in the Kuroshio and Gulf Stream Extension regions (e.g., Yang et al. 2017). The horizontal distribution of $\nabla \cdot \mathbf{Q}_{E}^{1}$ bears some similarity with that of $\Gamma_{K}^{0 \rightarrow 1}$ (Figs. 7d-f and Figs. 5a-c), indicating that $\nabla \cdot \mathbf{Q}_{E}^{1}$ acts to redistribute the mesoscale energy gained by flow instability in this region. When averaging over the large meander region, $\nabla \cdot \mathbf{Q}_{E}^{1}$ is negative (positive) for the tLM (NLM) state (Table 4). This means that there are more mesoscale energy transported into (out of) the considered domain during the tLM (NLM) state. On the synopticscale window, $\Gamma_{A}^{2}$ makes the most important source of energy (Table 5). During the tLM state, the energy budget is mostly balanced by the positive $\Gamma_{A}^{2}$ and negative $\Gamma_{K}^{2}$, indicating that high-frequency variabilities in this region are generated by baroclinic instability and then lose their energy back to lower-frequency flows through inverse KE cascades.

\section{Evolutions of the energetics during path transitions}

The previous section has provided a detailed description of the mixed instabilities and multiscale interactions among

TABLE 5. As in Table 2, but for the energy budget terms on the synoptic-scale window $(\varpi=2)\left(10^{-6} \mathrm{~m}^{3} \mathrm{~s}^{-3}\right)$.

\begin{tabular}{lrrrrr}
\hline \hline Term & $\partial E^{2} / \partial t$ & $\Gamma_{A}^{2}$ & \multicolumn{1}{c}{$\Gamma_{K}^{2}$} & $\nabla \cdot \mathbf{Q}_{E}^{2}$ & Residue \\
\hline tLM & -0.16 & 9.85 & -9.83 & -0.45 & -0.63 \\
nNLM & -0.05 & 2.12 & 2.59 & 0.53 & -4.23 \\
oNLM & 0.23 & 3.45 & 1.95 & 0.77 & -5.40 \\
\hline
\end{tabular}



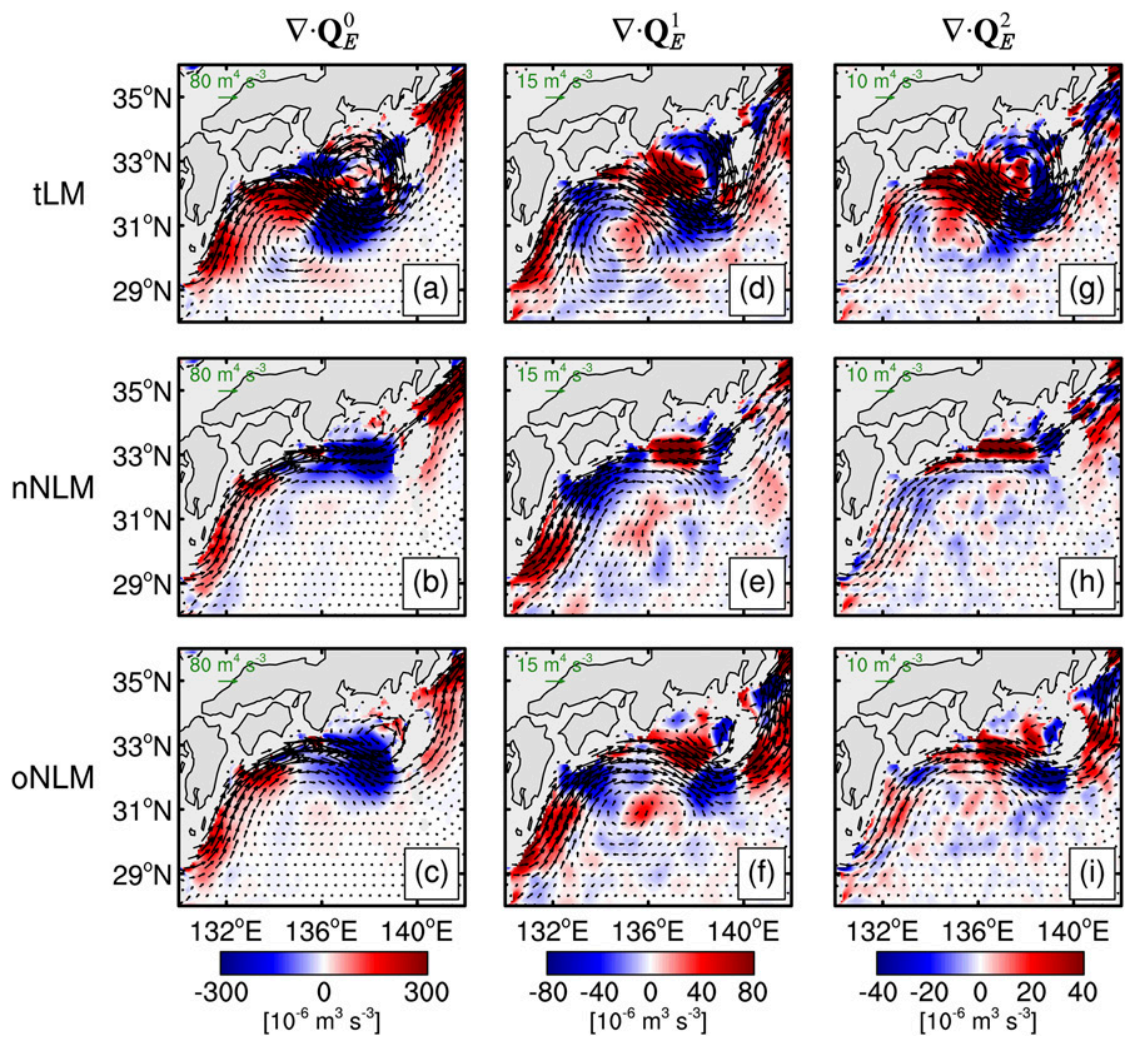

FIG. 7. As in Fig. 2, but for the transport $\left(\nabla \cdot \mathbf{Q}_{E}^{\varpi}\right.$; color shading $)$ and flux $\left(\mathbf{Q}_{E}^{\varpi}\right.$; vectors $)$ on the three scale windows.

the mean flow, mesoscale, and synoptic eddies from a composited point of view. In this section, we further examine the relevant time-dependent energetics underlying the Kuroshio path transitions, which is made possible by taking advantage of the temporal localization of the MWT (Liang and Anderson 2007). Only two major tLM events occurred since 1994. The first one occurred during 2004-05, which has so far received much attention in previous studies using satellite observations and numerical simulations (e.g., Miyazawa et al. 2008; Usui et al. 2008a,b, 2011). After 12 years since the 2004-05 tLM event, a new tLM is currently underway since August 2017 (Qiu 2019). In the following, we will diagnose the role of different instabilities, nonlocal transports, as well as inverse cascades on the path transitions regarding to these two tLM events.

Figure 8 shows the temporal evolution of the energetics averaged over the large meander region for the 2004-05 LM event. From Fig. 8b, it can be seen that a drastic increase of mesoscale energy $\left(E^{1}\right)$ occurs just before the LM formation. This feature is related to the arrival of a cyclonic eddy propagating from the upstream region (see the snapshots in the top row of Fig. 9), which has been believed as a triggering condition for the formation of the tLM path (Kawabe 1995; Miyazawa et al. 2004; Usui et al. 2008a; Liu et al. 2018). The origin of the $E^{1}$ burst is mostly nonlocal which can be confirmed in the energetic terms due to the complete separation of canonical transfer and transport in our energetics framework. It is found that the increase of $E^{1}$ is well correlated with a simultaneously increase of positive $-\nabla \cdot \mathbf{Q}_{E}^{1}$ (Fig. 8e); meanwhile, the baroclinic canonical transfer $\Gamma_{A}^{0 \rightarrow 1}$ exhibits positive but relatively small values (blue line in Fig. 8c). These results suggest that the growth of $E^{1}$ just before the LM formation is controlled by the cyclonic eddy propagating from the upstream region through energy advection, rather than local baroclinic instability process. Interestingly, the canonical transfer analysis shows that a significant negative barotropic transfer $\Gamma_{K}^{0 \rightarrow 1}$ appears just before the LM formation (blue line in Fig. 8d). This indicates that the incoming cyclonic eddy triggers an inverse KE cascade from the mesoscale window to the mean flow window, which helps to build up the KE of the LM.

After the LM is established, a significant increase of synoptic-scale energy $\left(E^{2}\right)$ occurs frequently throughout 
(a) Jet Position
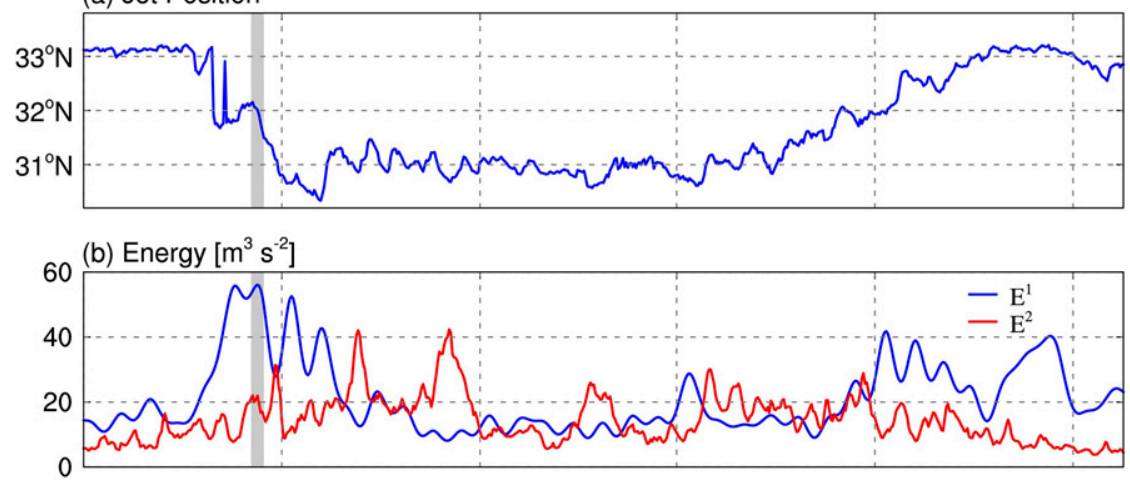

(c) Baroclinic Canonical Transfer $\left[10^{-6} \mathrm{~m}^{3} \mathrm{~s}^{-3}\right]$

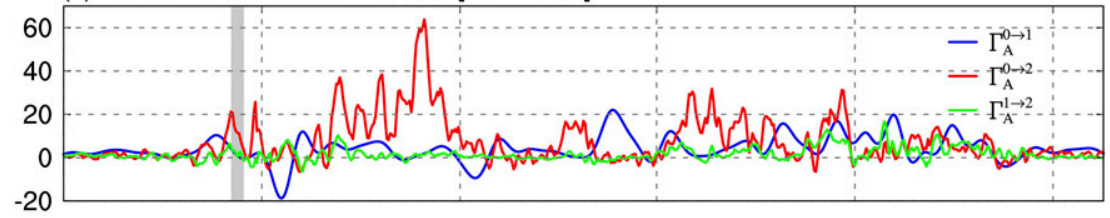

(d) Barotropic Canonical Transfer $\left[10^{-6} \mathrm{~m}^{3} \mathrm{~s}^{-3}\right]$

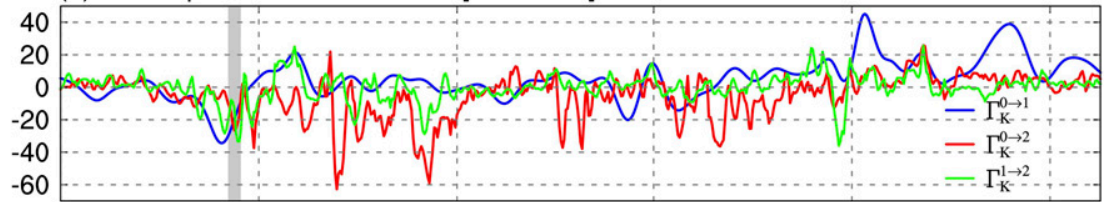

(e) Transport $\left[10^{-6} \mathrm{~m}^{3} \mathrm{~s}^{-3}\right]$

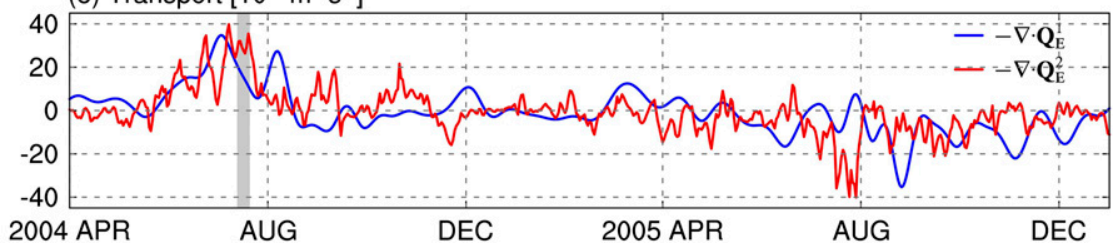

FIG. 8. Time series of the (a) jet position between $136^{\circ}$ and $139^{\circ} \mathrm{E}$; (b) $E^{1}$ (blue) and $E^{2}$ (red); (c) baroclinic transfers $\Gamma_{A}^{0 \rightarrow 1}$ (blue), $\Gamma_{A}^{0 \rightarrow 2}$ (red), and $\Gamma_{A}^{1 \rightarrow 2}$ (green); (d) barotropic transfers $\Gamma_{K}^{0 \rightarrow 1}$ (blue), $\Gamma_{K}^{0 \rightarrow 2}$ (red), and $\Gamma_{K}^{1 \rightarrow 2}$ (green); and (e) transport $-\nabla \cdot \mathbf{Q}_{E}^{1}$ (blue) and $-\nabla \cdot \mathbf{Q}_{E}^{2}$ (red) with sign reversed, averaged over the large meander region for the 2004-05 tLM event. The gray bar marks the beginning of the LM event.

the mature phase of the LM (red line in Fig. 8b). A movie of the SSH evolution in this high-frequency band clearly shows that these signals are dominated by small-scale frontal eddies propagating downstream along the background current. To illustrate, we plot the Hovmöller diagram of the synoptic-scale SSH along the Kuroshio jet axis which is defined by the 50 -cm contour as introduced in section 2 (Fig. 10b). It is obvious that the activities of these high-frequency frontal eddies are much enhanced during the LM state. In particular, when the Kuroshio meander is under well development (August 2004-April 2005), the frontal eddies around $140^{\circ} \mathrm{E}$ tend to be "blocked" by the LM rather than propagating downstream. The time series of area-mean $E^{2}$ is well correlated with that of the $\Gamma_{A}^{0 \rightarrow 2}$ (Figs. $8 \mathrm{~b}$ and $8 \mathrm{c}$ ), suggesting that the high-frequency, small-scale frontal eddies are mainly generated by baroclinic instability. More interestingly, it is found that the positive $\Gamma_{A}^{0 \rightarrow 2}$ is almost counterbalanced by negative $\Gamma_{K}^{0 \rightarrow 2}$ during the LM period (Fig. 10c). This means that when the LM is under development, high-frequency frontal eddies are more likely to be generated through extracting energy from the background meander via baroclinic instability, but the gained energy is given back to the meander through inverse KE cascades. At the same time, the area-mean energy transport keeps a relatively low value (Fig. 8e), providing a favorable environment for the inverse cascade to take its effect.

After August 2005 when the Kuroshio switches to the nNLM path, both $\Gamma_{A}^{0 \rightarrow 2}$ and $\Gamma_{K}^{0 \rightarrow 2}$ start to weaken. 

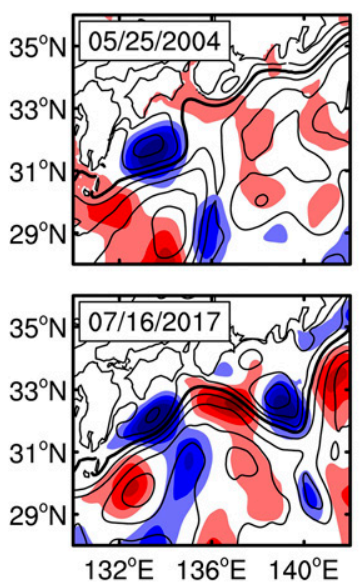
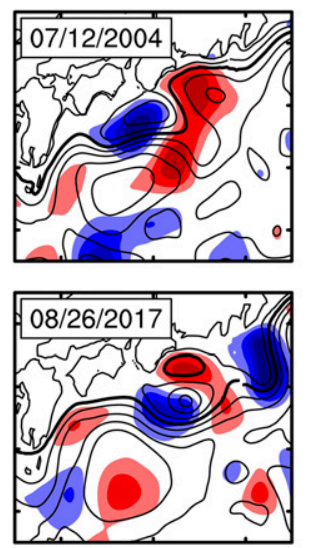

$132^{\circ} \mathrm{E} \quad 136^{\circ} \mathrm{E} \quad 140^{\circ} \mathrm{E}$
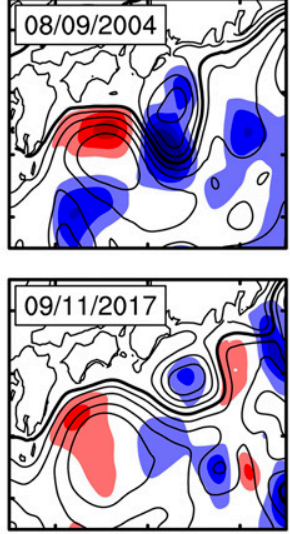

$132^{\circ} \mathrm{E} \quad 136^{\circ} \mathrm{E} \quad 140^{\circ} \mathrm{E}$
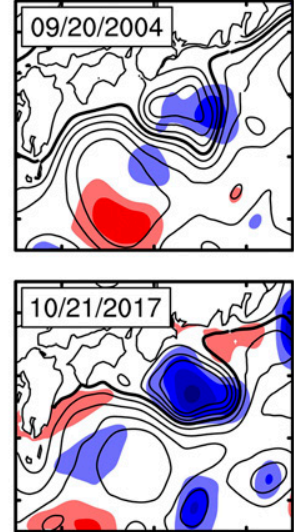

$132^{\circ} \mathrm{E} \quad 136^{\circ} \mathrm{E} \quad 140^{\circ} \mathrm{E}$
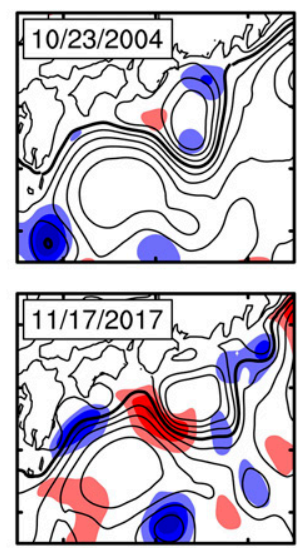

$132^{\circ} \mathrm{E} \quad 136^{\circ} \mathrm{E} \quad 140^{\circ} \mathrm{E}$

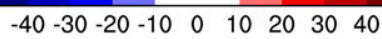

[cm]

FIG. 9. Daily snapshots of the mesoscale SSH (shaded; cm) for the (top) 2004-05 LM event and (bottom) 2017-present LM event from HYCOM. The original SSH field in each day is superposed in each figure, with the thick black line indicating the 50-cm contour. The contour interval is $10 \mathrm{~cm}$.

Note that there are another two episodes of $E^{1}$ growth during this LM decaying period, which is mainly due to barotropic instability (see the two positive $\Gamma_{K}^{0 \rightarrow 1}$ peaks in Fig. 8d). As for the nonlocal transport processes, both $-\nabla \cdot \mathbf{Q}_{E}^{1}$ and $-\nabla \cdot \mathbf{Q}_{E}^{2}$ show negative values during this period (Fig. 8e), indicating that the mechanical energies on the mesoscale and synoptic-scale windows are advected out of the large meander region.

To see whether the above dynamics is unique for the 2004-05 event, the above analysis is reconducted with respect to the 2017-present event. A noticeable feature of the 2017-present event is that, unlike the previous one which experiences a rapid growth of mesoscale energy $\left(E^{1}\right)$ just before the LM formation, the $E^{1}$ remains anomalously high for several months before the final establishment of the LM path (blue line in Fig. 11b). This is because that the Kuroshio path undergoes several short-term meanders (due to the downstream propagation of cyclonic eddies) since March 2017, and finally transits from an oNLM path to the tLM path in August 2017 (bottom row of Fig. 9). Although with such a difference, the dynamics underlying the two events seems to be quite similar. For example, the LM formation is preceded by a strong import of mesoscale energy carried by a cyclonic eddy propagating from upstream (Fig. 11e); a significant amount of KE cascades inversely from the mesoscale window to the mean flow window (Fig. 11d), and baroclinic instability is kept at a relatively low level during this period. The counterbalancing relation between the $\Gamma_{A}^{0 \rightarrow 2}$ and $\Gamma_{K}^{0 \rightarrow 2}$ is also at work after the formation of the LM. These results all suggest that the inverse $\mathrm{KE}$ cascades play an important role in the generation and maintenance of the Kuroshio large meander.

From the time series of $\Gamma_{A}^{0 \rightarrow 1}$ and $\Gamma_{K}^{0 \rightarrow 1}$, it is clear that baroclinic (barotropic) instability is more pronounced in the LM (NLM) state, both for the two tLM events (blue lines in Figs. 8c,d and Figs. 11c,d). Since the 2017present event is still under development, it is not clear whether the strong downscale KE transfer $\Gamma_{K}^{0 \rightarrow 1}$ episodes as revealed in the decaying state of the 2004-05 event is a universal feature. Finally, we address the time-dependent interaction between the mesoscale and synoptic eddies. The baroclinic canonical transfer $\Gamma_{A}^{1 \rightarrow 2}$ is generally small in magnitude (green lines in Figs. 8c and 11c); in contrast, the barotropic transfer $\Gamma_{K}^{1 \rightarrow 2}$ has a sizable magnitude, especially in the LM periods (green lines in Figs. 8d and 11d). Significant negative $\Gamma_{K}^{1 \rightarrow 2}$ is found just after strong $E^{1}$ episodes, indicating that the high-frequency frontal eddies not only pump energy to the mean flow, but also feed mesoscale motions with longer time scales than theirs.

\section{Conclusions}

In this study, we employed a recently developed multiscale energetics analysis and canonical transfer theory (Liang 2016) to investigate the dynamical processes related to the Kuroshio large meander south of Japan. Using a new analysis tool, namely, multiscale window transform (MWT), the related fields are reconstructed onto three scale windows, namely, the low-frequency mean flow window, mesoscale eddy 

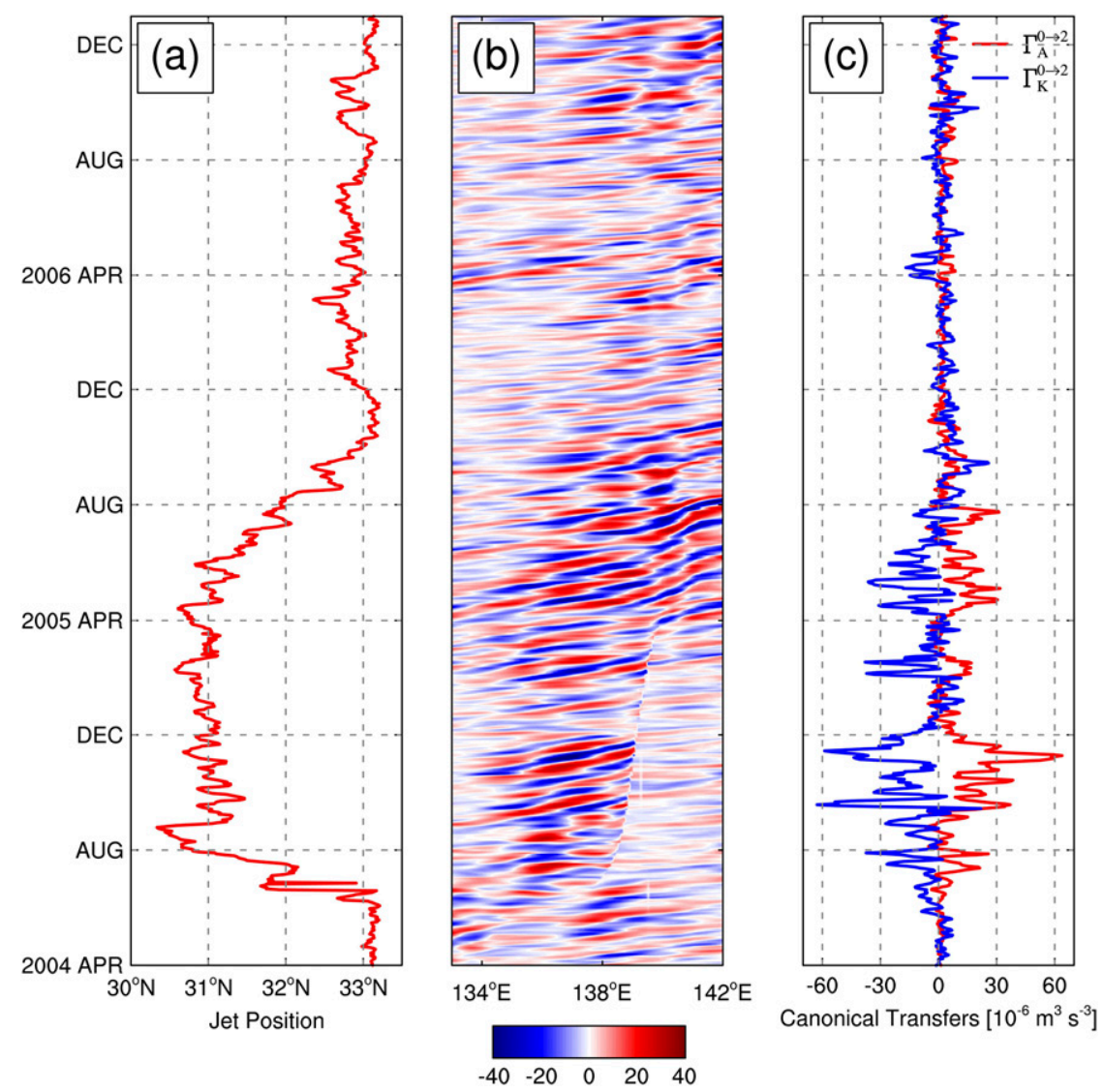

FIG. 10. (a) As in Fig. 8a. (b) Time-longitude plot of synoptic-scale SSH along the Kuroshio axis (defined by $50-\mathrm{cm} \mathrm{SSH}$ contour). The discontinuities around $140^{\circ} \mathrm{E}$ during August 2004 April 2005 are caused by the north-southward configuration of the jet axis along the eastern side of the large meander during this period. (c) Time series of $\Gamma_{A}^{0 \rightarrow 2}$ (red line) and $\Gamma_{K}^{0 \rightarrow 2}$ (blue line) averaged over the large meander region.

window, and high-frequency synoptic-scale window. The interactions between the scale windows are quantitatively evaluated in terms of canonical transfer, which bears a Lie bracket form and conserves energy in the space of scale. Three composites with reference to the typical Kuroshio path states south of Japan, that is, the tLM, nNLM, and oNLM path state, are first constructed, and the spatial patterns of the baroclinic and barotropic canonical transfers among the three scale windows for each composite state are presented. By investigating the canonical transfers between the mean flow and the mesoscale eddies, it is found that baroclinic (barotropic) instability is strengthened (weakened) during the tLM state, indicating that the increased mesoscale KE in the tLM state primarily arises from the baroclinic instability of the large meander. A new finding from our analysis is the overwhelming inverse cascade of KE during the tLM state from the synoptic eddies to the slowly varying mean flow, which occupies the whole large meander region. We emphasize that this is only identified for the tLM state, suggesting that the upscale energy transfer from the high-frequency variabilities is essential in maintaining the Kuroshio large meander. We also found that upscale KE transfer from the mesoscale eddies acts as another energy source for the large meander but is only limited to the eastern side of the meander trough.

By investigating the time-varying multiscale energetics underlying the 2004-05 and 2017-present tLM events, we found that the LM formations in both events are preceded by a strong import of mesoscale eddy energy carried by a cyclonic eddy propagating from upstream. The influx of mesoscale eddy energy triggers a significant inverse KE cascade from the mesoscale window to the mean flow window, helping to build up the KE reservoir for the large meander. After the LM is established, the synoptic frontal eddies are episodically intensified due to the baroclinic instability of the LM. But the gained energy is immediately fed back 

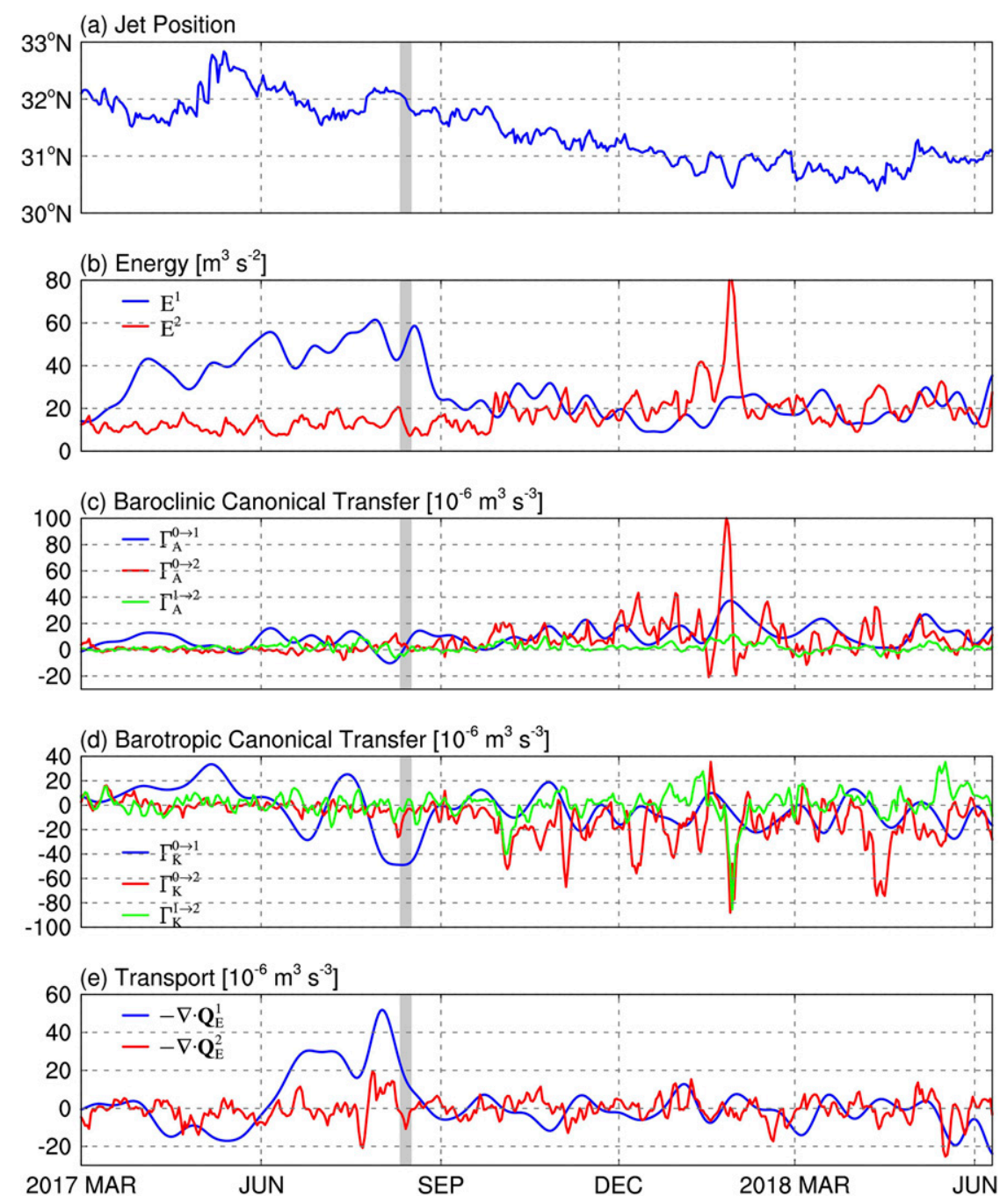

FIG. 11. As in Fig. 8, but for the 2017-present tLM event.

to the mean flow through inverse $\mathrm{KE}$ cascade. These results highlight the important role played by the inverse KE cascade in the generation and maintenance of the Kuroshio large meander.

We do not claim that the above findings apply to all the Kuroshio large meandering events south of Japan. Since there are only two tLM events occurring during the period of 1994-present, a longer simulation covering more LM events is needed to gain a more complete picture of the energetic scenario. Besides, a better resolution of the synoptic processes will help better understand the underlying multiscale dynamics. We will leave all that to future studies.

Acknowledgments. Thanks are due to two anonymous referees for their valuable suggestions. The HYCOM data are available at https://www.hycom.org/dataserver. In forming the first draft, Mingming Bi helped draw the figures. This work was supported by the National Science Foundation of
China (NSFC) under Grants 41806023 and 41276032, by the National Program on Global Change and Air-Sea Interaction (GASI-IPOVAI-06), by the 2015 Jiangsu Program of Entrepreneurship and Innovation Group, by Jiangsu Chair Professorship, by NUIST under Grant 2017r054, by the Natural Science Foundation of the Higher Education Institutions of Jiangsu Province (18KJB170019), and by the CSC-SOA Joint Scholarship Program (201804180031).

\section{APPENDIX}

\section{The Multiscale Window Transform and Canonical Transfer}

This appendix provides the needed information supplementary to section 3 about multiscale window transform (MWT) and multiscale energetics analysis. A detailed introduction of the methodology and theory is 
beyond the scope here. For that purpose, the reader is referred to Liang (2016) and Liang and Anderson (2007). A brief version similar to this one can also be seen in $\mathrm{Xu}$ and Liang (2017, section 2).

\section{a. Multiscale window transform}

As we know, the traditional Lorenz-type formalisms are formulated with temporal (or zonal) averaging and hence only result in energetics from a time-mean (or zonal mean) perspective, which are not suitable for highly localized and/or nonstationary processes (unfortunately, most oceanic processes are like this). To avoid the difficulty, a common practice is to use filters to retain the local information with respect to time (or space). However, how energy should be represented with the filtered fields becomes a fundamental problem, which, however, has been mostly overlooked. For example, a field $T(t)$ may be filtered into a background part $\bar{T}$ and an eddy part $T^{\prime}$, where both parts are time dependent. During the past decades, it has been a common practice to use $T^{2 / 2} / 2$ (i.e., with the time-averaging removed in the original Lorenztype formalism) as the energy for the eddy part, which is, unfortunately, conceptually wrong. To illustrate, suppose $T$ has a simple Fourier expansion with only two frequencies $\omega_{0}$ and $\omega_{1}$

$T=\underbrace{\left(a_{0} \cos \omega_{0} t+b_{0} \sin \omega_{0} t\right)}_{\bar{T}}+\underbrace{\left(a_{1} \cos \omega_{1} t+b_{1} \sin \omega_{1} t\right)}_{T^{\prime}}$,

where $\omega_{0} \ll \omega_{1}$ and the subscripts 0 and 1 represent the background (low frequency) and eddy (high frequency) processes, respectively. For this simple example, we know the energies for the background and eddy parts should be $\left(a_{0}^{2}+b_{0}^{2}\right) / 2$ and $\left(a_{1}^{2}+b_{1}^{2}\right) / 2$, respectively, that is, the square of the respective Fourier coefficients. They are absolutely not equal to the square of the reconstructed (filtered) fields, that is, $\bar{T}^{2} / 2$ and $T^{2} / 2$. That is to say, multiscale energy is a concept with the Fourier coefficients in phase space, which is related to its physical space counterpart through the Parseval equality in functional analysis (see Liang 2016 for details). In Eq. (A1), when $\bar{T}$ is a constant, it can be easily proved that $\left(a_{1}^{2}+b_{1}^{2}\right) / 2=\overline{T^{2}} / 2$, just as the eddy energy under the Reynolds decomposition in the Lorenz-type formalism. This explains why the time averaging operator in the Lorenz's energetics formalism cannot be removed, otherwise the resulting energy only bears the unit of energy, but does not make any sense in physics.

So it is by no means as trivial a problem as many people have been doing to obtain a physically consistent expression of time-dependent multiscale energy. In fact, this is a rather complex and fundamental problem and has been an impossible task until filter banks and wavelets are connected (Strang and Nguyen 1996). It has just been systematically addressed by Liang and Anderson (2007) in the development of MWT for this very purpose.

As mentioned above, application of a traditional filter yields reconstructed fields. These reconstructed fields are variables in physical space, hence cannot be used to represent energy. Liang and Anderson (2007) realized that, just as in the Fourier transform and inverse Fourier transform, there exists a transform-reconstruction pair for a class of specially devised orthogonal filters. (Note here orthogonality is crucial; otherwise the Parseval equality does not hold and hence energy cannot even be defined.) This pair is the very MWT and its counterpart, that is, multiscale window reconstruction (MWR). Generally, the MWR functions like what a traditional filter does, but the new transform coefficients from the MWT can be used to represent multiscale energy.

In brief, with the MWT, one can split a function space into a direct sum of several mutually orthogonal scale windows. For a time series $T(t)$, application of the MWT will yield MWT coefficients, which we write as $\hat{T}_{n}^{\sim \pi}$, where $\widehat{(\cdot)_{n}}$ denotes MWT on window $\varpi$ at time step $n$, and through MWR we obtain a reconstruction on window $\varpi$, written as $T^{\sim \varpi}(t)$. By a theorem called the property of marginalization, Liang and Anderson (2007) proved that the energy on window $\varpi$ is proportional to the square of the coefficients $\left(\hat{T}_{n}^{\sim \pi}\right)^{2} / 2$. Note it is by no means as trivial as $\left[T^{\sim \sigma}(t)\right]^{2} / 2$, which has frequently been seen in the literature.

\section{b. Canonical transfer}

Canonical transfer is a key concept in multiscale interaction; it is a faithful representation of the transfer process between scales or scale windows. The resulting measure of energy transfer is quite different from that in the traditional empirical formalisms. Originally canonical transfer was proposed by Liang and Robinson (2005) (called "perfect transfer" then), and was later rigorously proved by Liang (2016). To illustrate, consider a passive tracer $T$ in an incompressible flow $\mathbf{u}$ and neglect diffusion for simplicity,

$$
\frac{\partial T}{\partial t}+\nabla \cdot(\mathbf{u} T)=0
$$

Due to the existence of the nonlinear advection, multiscale interactions are expected to happen. Take MWT on both sides of Eq. (A2) and multiply $\hat{T}_{n}^{\sim \pi}$, an equation governing the energy evolution on scale window $\varpi$ results:

$$
\frac{\partial}{\partial t}\left[\frac{1}{2}\left(\hat{T}_{n}^{\sim \sigma}\right)^{2}\right]=-\hat{T}_{n}^{\sim \sigma} \nabla \cdot \widehat{(\mathbf{u} T)_{n}^{\sim \sigma}} .
$$


The term of the right-hand side of Eq. (A3) contains both transport and transfer processes. Transfer connects variabilities on different scales (e.g., the mean flow and transient eddies) and is hence related to the fundamental processes in geophysical fluid dynamics such as instabilities (Lorenz 1955; Harrison and Robinson 1978). So it is very important to have the two processes separated. However, this has been a continuing challenge. As has long been recognized, the existing empirical formalisms do not have a unique separation (Holopainen 1978; Plumb 1983). To tackle this problem, Liang (2016) proved, through reconstructing the "atomic" energy fluxes on the multiple scale windows, that a natural and unique separation exists. The resulting cross-scale transfer is

$$
\Gamma_{n}^{\varpi}=\frac{1}{2}\left[{\widehat{(\mathbf{u} T)_{n}}}^{\sim \pi} \cdot \nabla \hat{T}_{n}^{\sim \sigma}-\hat{T}_{n}^{\sim \pi} \nabla \cdot{\widehat{(\mathbf{u} T)_{n}}}^{\sim \pi}\right] .
$$

Readers are referred to Liang (2016, section 3) for detailed derivation. Notice that the resulting transfer bears a form similar to the Lie bracket and, particularly, the Poisson bracket in Hamiltonian mechanics. To see this, recall that a Poisson bracket $\{\cdot, \cdot\}$ is defined, for differential operators $(\partial / \partial x, \partial / \partial y)$ and functions $F$ and $G$, such that

$$
\{F, G\}=\frac{\partial F}{\partial y} \frac{\partial G}{\partial x}-\frac{\partial F}{\partial x} \frac{\partial G}{\partial y} .
$$

Consider the $1 \mathrm{D}$ version of $\Gamma_{n}^{\sigma}$, that is,

$$
\frac{1}{2}\left[{\widehat{(u T)_{n}}}_{n}^{\sim \sigma} \frac{\partial \hat{T}_{n}^{\sim \varpi}}{\partial x}-\hat{T}_{n}^{\sim \varpi} \frac{\partial \widehat{\left({ }_{u T}\right)_{n}^{\sim \sigma}}}{\partial x}\right] .
$$

If we pick two differential operators $(\partial / \partial x, \mathbf{I})$, where $\mathbf{I}$ is the identity, then the above canonical transfer is simply $\left\{\widehat{(u T)_{n},{ }^{\top}}, \hat{T}_{n}^{\sim \sigma}\right\} / 2$. Because of this, it has been termed "canonical transfer," in order to distinguish it from other transfers already existing in the literature. The canonical transfer expression naturally possesses the property as stated in Eq. (3), which means that it is a mere redistribution of energy among the scale windows, without generating or destroying energy as a whole. To compare canonical transfer to that resulting from the Lorenz-type formalism, Liang and Robinson (2007) considered the very special case with a Reynolds decomposition. In this case, the separated energy equations become

$$
\begin{aligned}
& \frac{\partial}{\partial t}\left(\frac{1}{2} \bar{T}^{2}\right)+\nabla \cdot\left(\frac{1}{2} \overline{\mathbf{u}} \bar{T}^{2}+\frac{1}{2} \bar{T} \overline{\mathbf{u}^{\prime} T^{\prime}}\right)=-\Gamma, \quad \text { and } \\
& \frac{\partial}{\partial t}\left(\frac{1}{2} \overline{T^{\prime 2}}\right)+\nabla \cdot\left(\frac{1}{2} \overline{\mathbf{u} T^{\prime 2}}+\frac{1}{2} \bar{T} \overline{\mathbf{u}^{\prime} T^{\prime}}\right)=\Gamma .
\end{aligned}
$$

where the second terms of the left-hand sides of Eqs. (A6) and (A7) are the in-scale transport process, and $\Gamma=(1 / 2)\left[\bar{T} \nabla \cdot\left(\overline{\mathbf{u}^{\prime} T^{\prime}}\right)-\overline{\mathbf{u}^{\prime} T^{\prime}} \cdot \nabla \bar{T}\right]$ represents the canonical transfer. Obviously, the right-hand side of Eqs. (A6) and (A7) is balanced and is hence a faithful representation of the energy transfer between the mean and eddy. In a traditional Lorenz-type framework, the energy equations for the mean and eddy fields are

$$
\begin{aligned}
& \frac{\partial}{\partial t}\left(\frac{1}{2} \bar{T}^{2}\right)+\nabla \cdot\left(\frac{1}{2} \overline{\mathbf{u}} \bar{T}^{2}\right)=-\bar{T} \nabla \cdot\left(\overline{\mathbf{u}^{\prime} T^{\prime}}\right), \quad \text { and } \\
& \frac{\partial}{\partial t}\left(\frac{1}{2} \overline{T^{\prime 2}}\right)+\nabla \cdot\left(\frac{1}{2} \overline{\mathbf{u} T^{\prime 2}}\right)=-\overline{\mathbf{u}^{\prime} T^{\prime}} \cdot \nabla \bar{T}
\end{aligned}
$$

respectively. One can see that the transfer terms on the right-hand side do not cancel out. The term $-\overline{\mathbf{u}^{\prime} T^{\prime}} \cdot \nabla \bar{T}$ is usually used to explain the energy source of eddy-mean flow interaction. Particularly, when $T$ is a velocity component, this term has been interpreted as the barotropic energy transfer extracted by Reynolds stress against the mean profile (e.g., Pedlosky 1987). Previously, Liang and Robinson (2007) illustrated, for a benchmark hydrodynamic instability model whose instability structure is analytically known, the traditional Reynolds stress extraction does not give the correct source of instability, while the canonical transfer $\Gamma$ does.

\section{REFERENCES}

Akitomo, K., T. Awaji, and N. Imasato, 1991: Kuroshio path variation south of Japan: 1. Barotropic inflow-outflow model. J. Geophys. Res., 96, 2549-2560, https://doi.org/10.1029/90JC02030.

_- M. Ooi, T. Awaji, and K. Kutsuwada, 1996: Interannual variability of the Kuroshio transport in response to the wind stress field over the North Pacific: Its relation to the path variation south of Japan. J. Geophys. Res., 101, 14 057-14 071, https://doi.org/10.1029/96JC01000.

Arbic, B. K., M. Müller, J. G. Richman, J. F. Shriver, A. J. Morten, R. B. Scott, G. Sérazin, and T. Penduff, 2014: Geostrophic turbulence in the frequency-wavenumber domain: Eddydriven low-frequency variability. J. Phys. Oceanogr., 44, 2050-2069, https://doi.org/10.1175/JPO-D-13-054.1.

Barnes, E. A., and D. L. Hartmann, 2010: Dynamical feedbacks and the persistence of the NAO. J. Atmos. Sci., 67, 851-865, https://doi.org/10.1175/2009JAS3193.1.

Berloff, P., A. M. C. Hogg, and W. Dewar, 2007: The turbulent oscillator: A mechanism of low-frequency variability of the wind-driven ocean gyres. J. Phys. Oceanogr., 37, 2363-2386, https://doi.org/10.1175/JPO3118.1.

Chao, S.-Y., 1984: Bimodality of the Kuroshio. J. Phys. Oceanogr., 14, 92-103, https://doi.org/10.1175/1520-0485(1984)014<0092: BOTK $>2.0 . \mathrm{CO} ; 2$.

Chassignet, E. P., H. E. Hurlburt, O. M. Smedstad, G. R. Halliwell, P. J. Hogan, A. J. Wallcraft, R. Baraille, and R. Bleck, 2007: The HYCOM (HYbrid Coordinate Ocean Model) data assimilative system. J. Mar. Syst., 65, 60-83, https://doi.org/ 10.1016/j.jmarsys.2005.09.016. 
Douglass, E. M., S. R. Jayne, F. O. Bryan, S. Peacock, and M. Maltrud, 2012: Kuroshio pathways in a climatologically forced model. J. Oceanogr., 68, 625-639, https://doi.org/10.1007/s10872012-0123-y.

Ebuchi, N., and K. Hanawa, 2000: Mesoscale eddies observed by TOLEX-ADCP and TOPEX/POSEIDON altimeter in the Kuroshio recirculation region south of Japan. J. Oceanogr., 56, 43-57, https://doi.org/10.1023/A:1011110507628.

Endoh, T., and T. Hibiya, 2001: Numerical simulation of the transient response of the Kuroshio leading to the large meander formation south of Japan. J. Geophys. Res., 106, $26833-$ 26 850, https://doi.org/10.1029/2000JC000776.

- and — 2009: Interaction between the trigger meander of the Kuroshio and the abyssal anticyclone over Koshu Seamount as seen in the reanalysis data. Geophys. Res. Lett., 36, L18604, https://doi.org/10.1029/2009GL039389.

- H. Tsujino, and T. Hibiya, 2011: The effect of Koshu Seamount on the formation of the Kuroshio large meander south of Japan. J. Phys. Oceanogr., 41, 1624-1629, https://doi.org/ 10.1175/JPO-D-11-074.1.

Gentile, V., S. Pierini, P. de Ruggiero, and L. Pietranera, 2018: Ocean modelling and altimeter data reveal the possible occurrence of intrinsic low-frequency variability of the Kuroshio Extension. Ocean Modell., 131, 24-39, https://doi.org/10.1016/ j.ocemod.2018.08.006.

Harrison, D. E., and A. R. Robinson, 1978: Energy analysis of open regions of turbulent flows - Mean eddy energetics of a numerical ocean circulation experiment. Dyn. Atmos. Oceans, 2 , 185-211, https://doi.org/10.1016/0377-0265(78)90009-X.

Holopainen, E. O., 1978: A diagnostic study of the kinetic energy balance of the long-term mean flow and the associated transient fluctuations in the atmosphere. Geophysica, 15, 125-145.

Hughes, R. L., 1989: The hydraulics of local separation in a coastal current with application to the Kuroshio meander. J. Phys. Oceanogr., 19, 1809-1820, https://doi.org/10.1175/1520-0485(1989) 019<1809:THOLSI $>2.0$. CO;2.

Hurlburt, H. E., A. J. Wallcraft, W. J. Schmitz, P. J. Hogan, and E. J. Metzger, 1996: Dynamics of the Kuroshio/Oyashio current system using eddy-resolving models of the North Pacific Ocean. J. Geophys. Res., 101, 941-976, https://doi.org/10.1029/ 95JC01674.

Kawabe, M., 1985: Sea level variations at the Izu Islands and typical stable paths of the Kuroshio. J. Oceanogr. Soc. Jpn, 41, 307326, https://doi.org/10.1007/BF02109238.

, 1995: Variations of current path, velocity, and volume transport of the Kuroshio in relation with the large meander. J. Phys. Oceanogr., 25, 3103-3117, https://doi.org/10.1175/ 1520-0485(1995)025<3103:VOCPVA > 2.0.CO;2.

Kurogi, M., and K. Akitomo, 2003: Stable paths of the Kuroshio south of Japan determined by the wind stress field. J. Geophys. Res., 108, 3332, https://doi.org/10.1029/2003JC001853.

- - H. Hasumi, and Y. Tanaka, 2013: Effects of stretching on maintaining the Kuroshio meander. J. Geophys. Res. Oceans, 118, 1182-1194, https://doi.org/10.1002/jgrc.20123.

Liang, X. S., 2016: Canonical transfer and multiscale energetics for primitive and quasigeostrophic atmospheres. J. Atmos. Sci., 73, 4439-4468, https://doi.org/10.1175/JAS-D-16-0131.1.

, and A. R. Robinson, 2005: Localized multiscale energy and vorticity analysis: I. Fundamentals. Dyn. Atmos. Oceans, 38, 195-230, https://doi.org/10.1016/j.dynatmoce.2004.12.004.

, and D. G. M. Anderson, 2007: Multiscale window transform. Multiscale Model. Simul., 6, 437-467, https://doi.org/10.1137/ 06066895X.
_ , and A. R. Robinson, 2007: Localized multi-scale energy and vorticity analysis: II. Finite-amplitude instability theory and validation. Dyn. Atmos. Oceans, 44, 51-76, https://doi.org/ 10.1016/j.dynatmoce.2007.04.001.

Liu, X., M. Mu, and Q. Wang, 2018: The nonlinear optimal triggering perturbation of the Kuroshio large meander and its evolution in a regional ocean model. J. Phys. Oceanogr., 48 , 1771-1786, https://doi.org/10.1175/JPO-D-17-0246.1.

Lorenz, E. N., 1955: Available potential energy and the maintenance of the general circulation. Tellus, 7, 157-167, https:// doi.org/10.3402/tellusa.v7i2.8796.

Luo, D., L. Zhong, and C. L. E. Franzke, 2015: Inverse energy cascades in an eddy-induced NAO-type flow: Scale interaction mechanism. J. Atmos. Sci., 72, 3417-3448, https://doi.org/ 10.1175/JAS-D-15-0062.1.

Ma, J., and X. S. Liang, 2017: Multiscale dynamical processes underlying the wintertime Atlantic blockings. J. Atmos. Sci., 74, 3815-3831, https://doi.org/10.1175/JAS-D-16-0295.1.

Masuda, A., 1982: An interpretation of the bimodal character of the stable Kuroshio path. Deep Sea Res., 29A, 471-484, https:// doi.org/10.1016/0198-0149(82)90071-1.

Masuda, S., and K. Akitomo, 2000: Effects of stratification and bottom topography on the Kuroshio path variation south of Japan. Part II: Path transitions in a multiple equilibrium regime. J. Phys. Oceanogr., 30, 1431-1449, https:// doi.org/10.1175/1520-0485(2000)030<1431:EOSABT $>$ 2.0.CO;2.

Metzger, E. J., and Coauthors, 2014: US Navy operational global ocean and Arctic ice prediction systems. Oceanography, 27, 32-43, https://doi.org/10.5670/oceanog.2014.66.

Mitsudera, H., B. Taguchi, T. Waseda, and Y. Yoshikawa, 2006: Blocking of the Kuroshio large meander by baroclinic interaction with the Izu Ridge. J. Phys. Oceanogr., 36, 20422059, https://doi.org/10.1175/JPO2945.1.

Miyazawa, Y., X. Guo, and T. Yamagata, 2004: Roles of mesoscale eddies in the Kuroshio paths. J. Phys. Oceanogr., 34, 2203-2222, https://doi.org/10.1175/1520-0485(2004)034<2203: ROMEIT $>2.0 . \mathrm{CO} ; 2$.

— T. Kagimoto, X. Guo, and H. Sakuma, 2008: The Kuroshio large meander formation in 2004 analyzed by an eddy-resolving ocean forecast system. J. Geophys. Res., 113, C10015, https:// doi.org/10.1029/2007JC004226.

Nagano, A., T. Hasegawa, H. Matsumoto, and K. Ariyoshi, 2018: Bottom pressure change associated with the 2004-2005 large meander of the Kuroshio south of Japan. Ocean Dyn., 68, 847865, https://doi.org/10.1007/s10236-018-1169-1.

O'Rourke, A. K., B. K. Arbic, and S. M. Griffies, 2018: Frequencydomain analysis of atmospherically forced versus intrinsic ocean surface kinetic energy variability in GFDL's CM2-O model hierarchy. J. Climate, 31, 1789-1810, https://doi.org/ 10.1175/JCLI-D-17-0024.1.

Pedlosky, J., 1987: Geophysical Fluid Dynamics. 2nd ed. SpringerVerlag, $710 \mathrm{pp}$

Pierini, S., 2006: A Kuroshio extension system model study: Decadal chaotic self-sustained oscillations. J. Phys. Oceanogr., 36, 1605-1625, https://doi.org/10.1175/JPO2931.1.

— H. A. Dijkstra, and M. Mu, 2014: Intrinsic low-frequency variability and predictability of the Kuroshio Current and of its extension. Adv. Oceanol. Limnol., 5, 79-122, https://doi.org/ 10.4081/aiol.2014.5352.

Plumb, R. A., 1983: A new look at the energy cycle. J. Atmos. Sci., 40, 1669-1688, https://doi.org/10.1175/1520-0469(1983)040<1669: ANLATE $>2.0 . \mathrm{CO} ; 2$. 
Qiu, B., 2019: Kuroshio and Oyashio currents. Encyclopedia of Ocean Sciences, J. K. Cochran, H. J. Bokuniewicz, and P. L. Yager, Eds., Vol. 3, 3rd ed. Elsevier, 384-394, https:// doi.org/10.1016/B978-0-12-409548-9.11295-3.

_ , and S. Chen, 2010: Eddy-mean flow interaction in the decadally modulating Kuroshio Extension system. Deep-Sea Res. II, 57, 1098-1110, https://doi.org/10.1016/j.dsr2.2008.11.036.

_- and W. Miao, 2000: Kuroshio path variations south of Japan: Bimodality as a self-sustained internal oscillation. J. Phys. Oceanogr., 30, 2124-2137, https://doi.org/10.1175/1520-0485(2000) 030<2124:KPVSOJ $>2.0 . \mathrm{CO} ; 2$.

Schmeits, M. J., and H. A. Dijkstra, 2001: Bimodal behavior of the Kuroshio and the Gulf Stream. J. Phys. Oceanogr., 31, 3435-3456, https://doi.org/10.1175/1520-0485(2001)031<3435: BBOTKA $>2.0 . \mathrm{CO} ; 2$.

Sekine, Y., 1990: A numerical experiment on the path dynamics of the Kuroshio with reference to the formation of the large meander path south of Japan. Deep Sea Res., 37A, 359-380, https://doi.org/10.1016/0198-0149(90)90014-M.

Sérazin, G., T. Penduff, B. Barnier, J.-M. Molines, B. K. Arbic, M. Müller, and L. Terray, 2018: Inverse cascades of kinetic energy as a source of intrinsic variability: A global OGCM study. J. Phys. Oceanogr., 48, 1385-1408, https://doi.org/ 10.1175/JPO-D-17-0136.1.

Solomon, H., 1978: Occurrence of small "trigger" meanders in the Kuroshio off southern Kyushu. J. Oceanogr., 34, 81-84, https:// doi.org/10.1007/BF02109256.

Strang, G., and T. Nguyen, 1996: Wavelets and Filter Banks. 2nd ed. Wellesley-Cambridge Press, 520 pp.

Sugimoto, S., and K. Hanawa, 2012: Relationship between the path of the Kuroshio in the south of Japan and the path of the Kuroshio Extension in the east. J. Oceanogr., 68, 219-225, https://doi.org/10.1007/s10872-011-0089-1.

Taft, B. A., 1972: Characteristics of the flow of the Kuroshio south of Japan. Kuroshio-Its Physical Aspects, H. Stommel and K. Yoshida, Eds., University of Tokyo Press, 165-216.

Tanaka, Y., and T. Hibiya, 2017: Effects of Koshu Seamount on the development of baroclinic instability leading to the Kuroshio large meander. J. Phys. Oceanogr., 47, 2563-2576, https:// doi.org/10.1175/JPO-D-17-0050.1.

Tseng, Y.-H., M.-L. Shen, S. Jan, D. E. Dietrich, and C.-P. Chiang, 2012: Validation of the Kuroshio current system in the dualdomain Pacific Ocean Model framework. Prog. Oceanogr. 105, 102-124, https://doi.org/10.1016/j.pocean.2012.04.003.

Tsujino, H., N. Usui, and H. Nakano, 2006: Dynamics of Kuroshio path variations in a high-resolution general circulation model J. Geophys. Res., 111, C11001, https://doi.org/10.1029/2005JC003118.

__ , S. Nishikawa, K. Sakamoto, N. Usui, H. Nakano, and G. Yamanaka, 2013: Effects of large-scale wind on the Kuroshio path south of Japan in a 60-year historical OGCM simulation. Climate Dyn., 41, 2287-2318, https://doi.org/ 10.1007/s00382-012-1641-4.

Tulloch, R., J. Marshall, C. Hill, and K. S. Smith, 2011: Scales, growth rates, and spectral fluxes of baroclinic instability in the ocean. J. Phys. Oceanogr., 41, 1057-1076, https://doi.org/ 10.1175/2011JPO4404.1.
Usui, N., H. Tsujino, Y. Fujii, and M. Kamachi, 2008a: Generation of a trigger meander for the 2004 Kuroshio large meander. J. Geophys. Res., 113, C01012, https://doi.org/10.1029/ 2008JE003225.

,,-- H. Nakano, and Y. Fujii, 2008b: Formation process of the Kuroshio large meander in 2004. J. Geophys. Res., 113, C08047, https://doi.org/10.1029/2008JE003225.

$[,-,-, \ldots$, and M. Kamachi, 2011: Decay mechanism of the 2004/05 Kuroshio large meander. J. Geophys. Res., 116, C10010, https://doi.org/10.1029/2011JC007009.

von Storch, J.-S., C. Eden, I. Fast, H. Haak, D. Hernández-Deckers, E. Maier-Reimer, J. Marotzke, and D. Stammer, 2012: An estimate of the Lorenz energy cycle for the World Ocean based on the STORM/NCEP simulation. J. Phys. Oceanogr., 42, 21852205, https://doi.org/10.1175/JPO-D-12-079.1.

Wang, Q., M. Mu, and H. A. Dijkstra, 2013: Effects of nonlinear physical processes on optimal error growth in predictability experiments of the Kuroshio Large Meander. J. Geophys. Res. Oceans, 118, 6425-6436, https://doi.org/ 10.1002/2013JC009276.

Waseda, T., H. Mitsudera, B. Taguchi, and Y. Yoshikawa, 2003: On the eddy-Kuroshio interaction: Meander formation process. J. Geophys. Res., 108, 3220, https://doi.org/10.1029/ 2002JC001583.

$\longrightarrow,-,-$, and K. Kutsuwada, 2005: Significance of highfrequency wind forcing in modelling the Kuroshio. J. Oceanogr., 61, 539-548, https://doi.org/10.1007/s10872-005-0061-z.

Wunsch, C., P. Heimbach, R. Ponte, and I. Fukumori, 2009: The global general circulation of the ocean estimated by the ECCO-Consortium. Oceanography, 22, 88-103, https:// doi.org/10.5670/oceanog.2009.41.

Xu, F., and X. S. Liang, 2017: On the generation and maintenance of the 2012/13 sudden stratospheric warming. J. Atmos. Sci., 74, 3209-3228, https://doi.org/10.1175/JAS-D-17-0002.1.

Yamagata, T., and S.-I. Umatani, 1989: Geometry-forced coherent structures as a model of the Kuroshio large meander. J. Phys. Oceanogr., 19, 130-138, https://doi.org/10.1175/1520-0485(1989) 019<0130:GFCSAA > 2.0.CO;2.

Yang, Y., and X. S. Liang, 2016: The instabilities and multiscale energetics underlying the mean-interannual-eddy interactions in the Kuroshio Extension region. J. Phys. Oceanogr., 46, 14771494, https://doi.org/10.1175/JPO-D-15-0226.1.

- and - 2019: The intrinsic nonlinear multiscale interactions among the mean flow, low frequency variability and mesoscale eddies in the Kuroshio region. Sci. China Earth Sci., 62, 595-608, https://doi.org/10.1007/s11430-018-9289-4.

,,$-- B$. Qiu, and S. Chen, 2017: On the decadal variability of the eddy kinetic energy in the Kuroshio Extension. J. Phys. Oceanogr., 47, 1169-1187, https://doi.org/10.1175/JPO-D-16-0201.1.

Yasuda, I., J.-H. Yoon, and N. Suginohara, 1985: Dynamics of the Kuroshio large meander. J. Oceanogr. Soc. Japan, 41, 259-273, https://doi.org/10.1007/BF02109275.

Yoon, J.-H., and I. Yasuda, 1987: Dynamics of the Kuroshio large meander: Two-layer model. J. Phys. Oceanogr., 17, 66-81, https://doi.org/10.1175/1520-0485(1987)017<0066: DOTKLM $>2.0 . \mathrm{CO} ; 2$. 